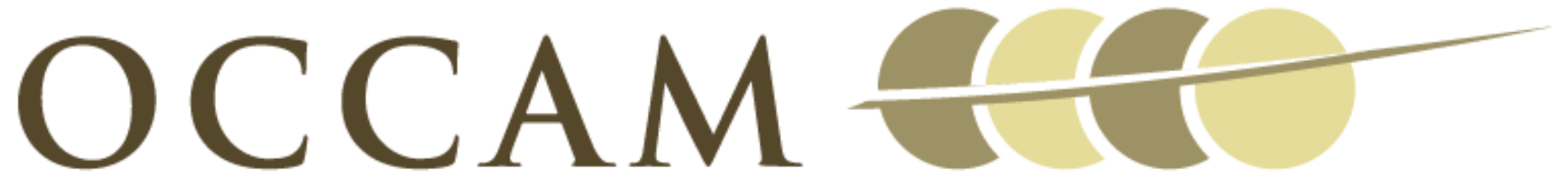

OXFORD CENTRE FOR COLLABORATIVE APPLIED MATHEMATICS

Report Number 10/12

Nonlinear Morphoelastic Plates I: Genesis of Residual Stress

by

Joseph McMahon, Alain Goriely, and Michael Tabor

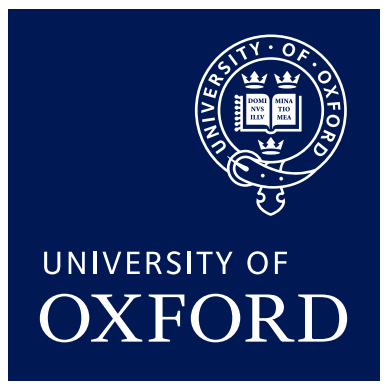

Oxford Centre for Collaborative Applied Mathematics Mathematical Institute 24 - 29 St Giles'

Oxford

OX1 3LB

England 



\section{Nonlinear Morphoelastic Plates I: Genesis of Residual Stress}

\section{Joseph McMahon*, Alain Goriely*i‡, and Michael Tabor ${ }^{* \dagger}$}

${ }^{*}$ Program in Applied Mathematics and ${ }^{\dagger}$ Department of Mathematics,

University of Arizona, Building \#89, Tucson, AZ 85721

${ }^{\dagger}$ OCCAM, Institute of Mathematics, University of Oxford, UK;

email: goriely@maths.ox.ac.uk, Corresponding author: A. G.

Abstract Volumetric growth of an elastic body may give rise to residual stress. Here a rigorous analysis of the residual strains and stresses generated by growth in the axisymmetric Kirchhoff plate is given. Balance equations are derived via the global constraint principle, growth is incorporated via a multiplicative decomposition of the deformation gradient, and the system is closed by a response function. The particular case of a compressible neo-Hookean material is analyzed and the existence of residually stressed states is established.

KEYWORDS: Nonlinear elasticity, Kirchhoff Plates, Growth, Residual Stress. 


\section{Contents}

1 Introduction $r$

2 Kinematics $\quad 3$

2.1 Deformation and Deformation Gradient . . . . . . . . . . . . . . . 4

2.2 Incompatible Growth . . . . . . . . . . . . . . . . . . . . . . . . . . . . . . . . .

2.3 Elastic Response . . . . . . . . . . . . . . . . . . . . . . 6

2.4 Explicit Expressions . . . . . . . . . . . . . . . . . . . 7

3 Equation of Mechanical Equilibrium $\quad 8$

3.1 The Global Constraint Principle . . . . . . . . . . . . . . . . . . 8

3.2 Cylindrical Deformations of the Axisymmetric Plate . . . . . . . . . . . . 8

4 Neo-Hookean Constitutive Relation 10

5 Conversion to a Dynamical System 12

6 No Radial Growth: $\gamma_{1}(R) \equiv 1 \quad 14$

6.1 Fixed Points and Linearization . . . . . . . . . . . . . . . . . . . 15

6.2 Existence of Solution . . . . . . . . . . . . . . . . . . . . 16

6.3 Numerical Results. . . . . . . . . . . . . . . . . . . . 20

7 Constant Radial Growth: $\gamma_{1}(R) \equiv \gamma_{0}>1 \quad 20$

8 Conclusion $\quad 23$

\section{Introduction}

The description of volumetric growth of an elastic material within the framework of continuum mechanics can be achieved through the multiplicative decomposition of the deformation gradient [23]. The basic idea is to decompose the growth process and the elastic response in two different deformation steps. A volumetric growth deformation tensor is specified locally and is followed by an elastic response ensuring integrity and continuity of the body as well as specific boundary conditions. However, the local specification of volumetric growth of a three-dimensional body may distort the constituent volume elements in such a way that the grown elements are unable to form a continuous body residing in Euclidean space. This situation is usually referred to as incompatible growth and corresponds to a growth deformation tensor which cannot be expressed as the gradient of a vector field. Geometrically speaking this means that the set of distances between points in the grown body does not agree with the set of Euclidean distances between points in any simply connected subset of three-dimensional Euclidean space. However, if an elastic response distorts the grown volume elements so that they again form a continuous Euclidean body this can give rise to so called residual stresses, that is stresses that remain in the body in the absence of loading. Residual stresses are found in many soft tissues and are of particular importance in a number of bio-mechanical contexts, e.g. in the genesis of stress in blood vessels and trachea $[6,7,10,13,25,24,2]$. The elastic 
responses to growth and the stability thereof have been studied in depth for three-dimensional models of growing elastic bodies with spherical shell [14, 1] and cylindrical geometries [15, 26].

Many biological structures such as leaves, skin, and thin biological membranes are essentially two dimensional. Therefore, it is of interest to adapt the theory of volumetric growth to structures which can be described by two dimensional elasticity. Previous works along these lines include: the analysis of cavitation in growing elastic membranes [20]; the development of a theory (linear) elastic plates equipped a priori with metric tensors characterizing growth and such that preclude their isometric immersion into three-dimensional Euclidean space [12]; and the generalization of Föppl-von Kármán equation to include an extra source of mean curvature [11]. Our approach here is different in the sense that we do not use the assumption that the body is thin. Rather, following the theory of Cosserat and Kirchhoff plates, we constrain the possible deformations of a three-dimensional body to deformations compatible with the twodimensional geometry [3]. This approach allows to explore the full nonlinear behavior of the material and does not restrict the analysis to thin bodies.

The plan of the paper is as follows: first we examine the kinematics of cylindrical deformations of an axisymmetic plate and offer two interpretations of the multiplicative decomposition of the deformation gradient. In Section 3 the Global Constraint Principle is applied to derive balance equations for a plate constrained to a class of "plate-like" configurations. A nonlinearly elastic constitutive relation is chosen in Section 4 and is modified to model the elastic distortion from an incompatibly grown state. In Section 5 the closed system of ordinary differential equations is converted to a set of autonomous equations. In Sections 6 and 7 dynamical systems theory is employed to establish the existence of solutions of the equations for two classes of growth tensors. Results of numerical experiments are also presented. In this paper we study the Kirchhoff plate, namely one that is constrained to maintain constant thickness; however, a similar model allowing for expansion or contraction through the thickness can be derived through a similar procedure.

\section{Kinematics}

By Euclidean 3-space we mean the space $\mathbb{E}^{3}$ of space points equipped with the inner product:

$$
\left(x_{1} \boldsymbol{i}+y_{1} \boldsymbol{j}+z_{1} \boldsymbol{k}\right) \cdot\left(x_{2} \boldsymbol{i}+y_{2} \boldsymbol{j}+z_{2} \boldsymbol{k}\right)=x_{1} x_{2}+y_{1} y_{2}+z_{1} z_{2} .
$$

This endows each spatial point with a norm,

$$
\|x \boldsymbol{i}+y \boldsymbol{j}+z \boldsymbol{k}\|=\sqrt{x^{2}+y^{2}+z^{2}},
$$

which indicates the distance of that point from the origin.

To each point of $\mathbb{E}^{3}$ there is anchored a three-dimensional vector space of tangent vectors. Each such vector space is called the tangent space at that point in $\mathbb{E}^{3}$. Other than spatial position in $\mathbb{E}^{3}$, all vectorial and tensorial quantities discussed, such as forces and deformation gradients, will be constructed from tangent vectors and linear functionals on tangent vectors. Since each tangent space is a distinct vector space, each has a distinct local basis of vectors that span it. 
For example, for the cylindrical coordinates that will be used here, we have the local orthonormal basis

$$
\boldsymbol{e}_{1}(\Theta)=\cos \Theta \boldsymbol{i}+\sin \Theta \boldsymbol{j}, \quad \boldsymbol{e}_{2}(\Theta)=-\sin \Theta \boldsymbol{i}+\cos \Theta \boldsymbol{j}, \quad \text { and } \quad \boldsymbol{k} .
$$

See Figure 1.

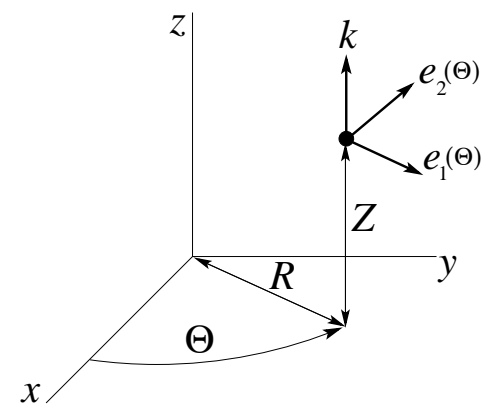

Figure 1: Cylindrical coordinates and local orthonormal basis

We use these vectors to define the position function for the reference configuration:

$$
\boldsymbol{X}(R, \Theta, Z)=R \boldsymbol{e}_{1}(\Theta)+Z \boldsymbol{k} .
$$

When used to define a position function, this linear combination of vectors may be considered as a tangent vector anchored at the origin of $\mathbb{E}^{3}$ and pointing to the spatial position occupied by the material point with coordinates $(R, \Theta, Z)$. When used in expressions for force and stress at a spatial point, however, they are to be understood as tangent vectors anchored at the spatial point in question, as shown in Figure 1.

\subsection{Deformation and Deformation Gradient}

If we let $\mathcal{B}$ be the set of coordinates $(R, \Theta, Z)$ of all the material points of the body, then $\boldsymbol{X}(\mathcal{B})$ is the subset of $\mathbb{E}^{3}$ occupied by the body in its reference configuration. A deformation of the body is a differentiable, invertible map $\chi: \boldsymbol{X}(\mathcal{B}) \rightarrow \mathbb{E}^{3}$ whose value $\boldsymbol{x}_{0}=\boldsymbol{\chi}\left(\boldsymbol{X}_{0}\right)$ is the spatial position of the material point originally located at $\boldsymbol{X}_{0} \in \boldsymbol{X}(\mathcal{B})$.

A local, linear description of the deformation is provided by its gradient. This deformation gradient maps infinitesimal material lines in the reference configuration to their images in the deformed configuration. Since a material line is a tangent vector, this makes the deformation gradient a two-point tensor: it maps tangent vectors anchored at a point $\boldsymbol{X}_{0}$ in the reference configuration to tangent vectors anchored at $\boldsymbol{x}_{0}=\boldsymbol{\chi}\left(\boldsymbol{X}_{0}\right)$, the image of $\boldsymbol{X}_{0}$ under the deformation.

If the body is deformed so that it remains cylindrical and is neither stretched nor compressed along the vertical direction, the material point originally located at $\boldsymbol{X}(R, \Theta, Z)$ has the new location

$$
\boldsymbol{x}(R, \Theta, Z)=r(R) \boldsymbol{e}_{1}(\Theta)+Z \boldsymbol{k} .
$$


A tangent space anchored at a point in the reference configuration has a local basis formed by the derivatives

$$
\frac{\partial \boldsymbol{X}}{\partial R}=\boldsymbol{e}_{1}, \quad \frac{\partial \boldsymbol{X}}{\partial \Theta}=R \boldsymbol{e}_{2}, \quad \text { and } \quad \frac{\partial \boldsymbol{X}}{\partial Z}=\boldsymbol{k} .
$$

At the image under the deformation, the images of these derivatives are

$$
\frac{\partial \boldsymbol{x}}{\partial R}=r^{\prime} \boldsymbol{e}_{1}, \quad \frac{\partial \boldsymbol{x}}{\partial \Theta}=r \boldsymbol{e}_{2}, \quad \text { and } \quad \frac{\partial \boldsymbol{x}}{\partial Z}=\boldsymbol{k}
$$

The deformation gradient, which we label $\boldsymbol{F}$, should have the following dot-products with the local basis vectors in the reference configuration:

$$
\begin{aligned}
\boldsymbol{F} \cdot \boldsymbol{e}_{1} & =r^{\prime} \boldsymbol{e}_{1} \\
\boldsymbol{F} \cdot\left(R \boldsymbol{e}_{2}\right) & =r \boldsymbol{e}_{2}, \\
\boldsymbol{F} \cdot \boldsymbol{k} & =\boldsymbol{k}
\end{aligned}
$$

Hence, the full deformation gradient is

$$
\boldsymbol{F}=r^{\prime} \boldsymbol{e}_{1} \otimes \boldsymbol{e}_{1}+\frac{r}{R} \boldsymbol{e}_{2} \otimes \boldsymbol{e}_{2}+\boldsymbol{k} \otimes \boldsymbol{k}
$$

where the right vector in each tensor product is to be understood as a tangent vector anchored at a point in the reference configuration, while the left vector is a tangent vector anchored at a point in the final, deformed configuration.

\subsection{Incompatible Growth}

The very special relationship between the coefficients of $\boldsymbol{e}_{1} \otimes \boldsymbol{e}_{1}$ and $\boldsymbol{e}_{2} \otimes \boldsymbol{e}_{2}$ in $\boldsymbol{F}$ reflects the fact that not all tensor fields of this form are equal to deformation gradients. Consider, for example,

$$
\boldsymbol{G}(R, \Theta, Z)=\gamma_{1}(R) \boldsymbol{e}_{1}(\Theta) \otimes \boldsymbol{e}_{1}(\Theta)+\gamma_{2}(R) \boldsymbol{e}_{2}(\Theta) \otimes \boldsymbol{e}_{2}(\Theta)+\boldsymbol{k} \otimes \boldsymbol{k}
$$

If $\boldsymbol{G}$ is the gradient of a cylindrical deformation of the form considered above, then $\gamma_{1}=r^{\prime}$ and $\gamma_{2}=r / R$, which implies

$$
\gamma_{1}=\frac{d}{d R}\left(R \gamma_{2}\right)
$$

If this is not satisfied, then $\boldsymbol{G}$ is not a deformation gradient of the type considered above. In fact, computing the Riemann curvature tensor (see Chapter 1 of [9]) that arises from $\boldsymbol{G}$ and observing that it does not vanish identically shows that $\boldsymbol{G}$ is not the gradient of any deformation. A two-point tensor field that is not equal to a deformation gradient will be called incompatible.

Each fixed tensor with positive determinant is equal to the local value of some deformation gradient; it is only in the failure of local values to "patch" together correctly that a non-gradient differs from a deformation gradient. Incompatible tensor fields have been used for decades in the theory of elastoplasticity $[17,18,27]$, where $\boldsymbol{F}$ is decomposed into the product of two incompatible tensor fields:

$$
\boldsymbol{F}=\boldsymbol{A} \cdot \boldsymbol{G}
$$


This multiplicative decomposition has more recently been employed in elastic models of growing solids [23]. In this context, $\boldsymbol{G}$ describes incompatible volumetric growth. A local value of $\boldsymbol{G}$ describes the deformation that a growing element of matter would undergo if it were not constrained by the presence of neighboring matter. The grown elements are considered stressfree, but they no longer form a continuous solid. The tensor field $\boldsymbol{A}$ plays the rôle of the elastic deformation needed to reestablish a continuous solid. However, since $\boldsymbol{G}$ is not compatible and $\boldsymbol{F}$ is, $\boldsymbol{A}=\boldsymbol{F} \cdot \boldsymbol{G}^{-1}$ is not a deformation gradient. Thus the step from the grown, stress-free state to the final state, which may carry residual stress even in the absence of applied loads and body forces, cannot properly be called an elastic deformation. This is illustrated in Figure 2.

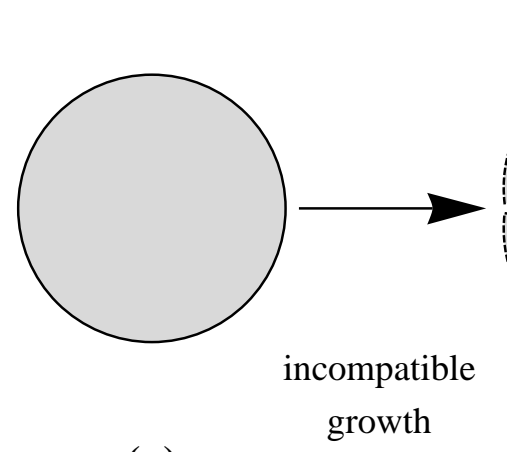

(a)

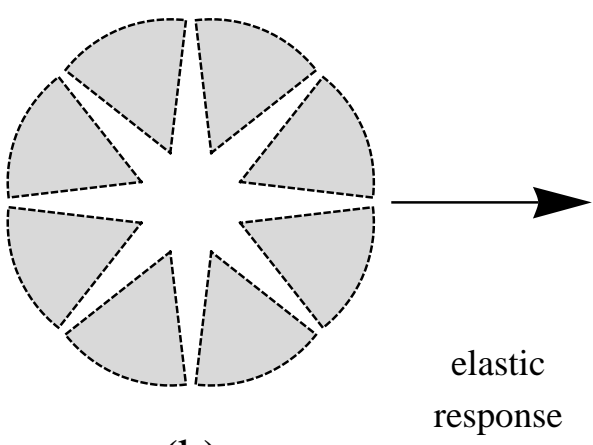

(b) response

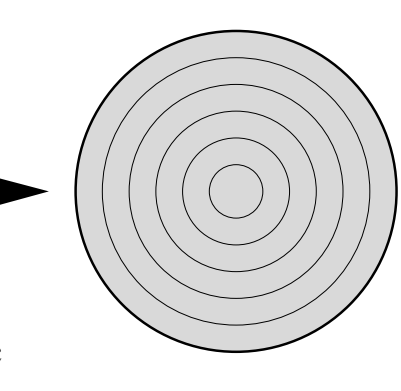

(c)

Figure 2: In (a), the body is in its reference configuration. Incompatible growth precludes stress-free grown elements of matter (b) from forming a continuous body. The body's elastic response determines how the grown elements are distorted to form a continuous body again (c). This body will carry residual stress.

However, there is an alternative interpretation in which the body remains a continuous manifold, avoiding concerns over the energetics of the tearing-apart and re-assembly of the body, as examined in [16]. If we let the incompatible growth tensor $\boldsymbol{G}$ change the metric tensor, then the arclengths between points in the body preclude the body "fitting" into $\mathbb{E}^{3}$. The points of the body still form a continuous three-dimensional manifold with the growth metric defined by $\boldsymbol{G}^{\mathrm{T}} \cdot \boldsymbol{G}$ but, it is a manifold that cannot be isometrically immersed in $\mathbb{E}^{3}$.

A two-dimensional analogue of this is illustrated in Figure 3. A flat disc has its metric tensor changed by incompatible growth, resulting in a two-dimensional manifold that can no longer fit into the Euclidean plane. The subsequent elastic response restores the body's isometric immersibility into $\mathbb{E}^{2}$, but the resulting disc carries residual stress, even in the absence of applied loads and body forces.

\subsection{Elastic Response}

There is no unique tensor field $\boldsymbol{A}$ that renders the product $\boldsymbol{A} \cdot \boldsymbol{G}$ a deformation gradient. For example, if $\boldsymbol{A}=\boldsymbol{G}^{-1}$, then the product is $\boldsymbol{A} \cdot \boldsymbol{G}=\boldsymbol{G}^{-1} \cdot \boldsymbol{G}=\boldsymbol{I}$, which is the gradient of the identity deformation $\boldsymbol{X} \mapsto \boldsymbol{X}$. We will seek a particular $\boldsymbol{A}$ by assuming that the grown body has a hyperelastic constitutive relation. As shown in $[8,1]$, a hyperelastic constitutive relation 


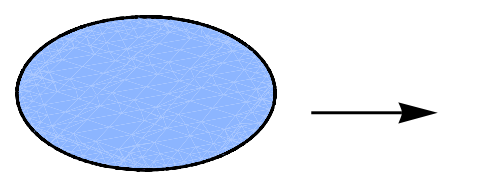

(a)

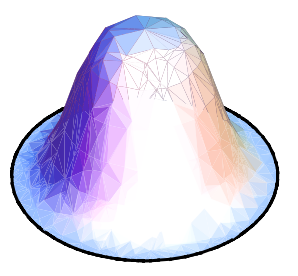

(b)

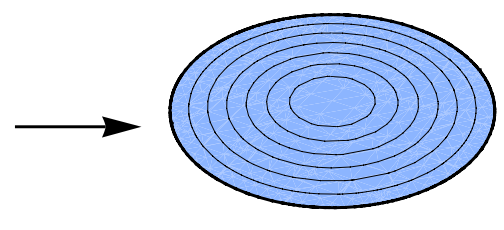

(c)

Figure 3: An interpretation of the multiplicative decomposition that avoids tearing the body into pieces. In this two-dimensional analogue, the flat disc (a) is given a new metric tensor so that its arclengths preclude its remaining in the Euclidean plane. It is viewed as a nonEuclidean surface in (b). After the elastic response, the body is again a disc in the Euclidean plane (c) but carries residual stress.

should recognize only the elastic portion of the deformation gradient. Hence, we assume that the Cauchy stress tensor has the form

$$
\boldsymbol{T}(\boldsymbol{x})=\widehat{\boldsymbol{T}}(\boldsymbol{A}(\boldsymbol{x})),
$$

where $\widehat{\boldsymbol{T}}$ is the gradient of some scalar energy density. Our derivation of balance laws refers to the reference and final configurations and, accordingly, the stress used here is the first PiolaKirchhoff stress tensor:

$$
\boldsymbol{P}=(\operatorname{det} \boldsymbol{F}) \boldsymbol{T} \cdot \boldsymbol{F}^{-1} .
$$

\subsection{Explicit Expressions}

We will need explicit expressions for several quantities in calculations. First among these is $\boldsymbol{A}$, the elastic factor of $\boldsymbol{F}$ :

$$
\boldsymbol{A}=\boldsymbol{F} \cdot \boldsymbol{G}^{-1}=\frac{r^{\prime}}{\gamma_{1}} \boldsymbol{e}_{1} \otimes \boldsymbol{e}_{1}+\frac{r}{\gamma_{2} R} \boldsymbol{e}_{2} \otimes \boldsymbol{e}_{2}+\boldsymbol{k} \otimes \boldsymbol{k} .
$$

Since $\boldsymbol{A}$ is not a deformation gradient, its eigenvalues are not true "stretches". We choose the label pseudostretches for the eigenvalues of $\boldsymbol{A}$; they are

$$
\lambda_{1}=\frac{r^{\prime}}{\gamma_{1}}, \quad \lambda_{2}=\frac{r}{\gamma_{2} R}, \quad \lambda_{3}=1 .
$$

We also will make reference to

$$
\boldsymbol{F}^{-T}=\frac{1}{r^{\prime}} \boldsymbol{e}_{1} \otimes \boldsymbol{e}_{1}+\frac{R}{r} \boldsymbol{e}_{2} \otimes \boldsymbol{e}_{2}+\boldsymbol{k} \otimes \boldsymbol{k} .
$$




\section{Equation of Mechanical Equilibrium}

\subsection{The Global Constraint Principle}

The mechanical equations of thin plates can be derived by viewing the plate as either a twodimensional body with a "director field" attached to it or a three-dimensional body subject to constraints that keep it in a "plate-like" configuration. The latter approach yields "induced" theories of plates in which constitutive restrictions are added to the full three-dimensional theory [3].

When we view the plate as a constrained three-dimensional body, we consider a class of allowed deformations $\boldsymbol{X} \mapsto \boldsymbol{x}$. Further, we assume that the first Piola-Kirchhoff stress tensor $\boldsymbol{P}$ can be decomposed into a sum of an "active" stress $\boldsymbol{P}_{\text {act }}$ and a "latent" stress $\boldsymbol{P}_{\text {lat }}[4,19]$ :

$$
\boldsymbol{P}=\boldsymbol{P}_{a c t}+\boldsymbol{P}_{l a t},
$$

where $\boldsymbol{P}_{\text {act }}$ is determined by a constitutive relation involving the deformation gradient $\partial \boldsymbol{x} / \partial \boldsymbol{X}$ and

$$
\int_{\mathcal{B}} \boldsymbol{P}_{l a t}: \frac{\partial \stackrel{\Delta}{\boldsymbol{x}}}{\partial \boldsymbol{X}} d v=0
$$

for each "virtual displacement" $\stackrel{\Delta}{\boldsymbol{x}}$ tangent to the manifold of allowed configurations, where $\mathcal{B}$ is the reference configuration of the body; see below for details. $\boldsymbol{P}_{l a t}$ is that portion of the stress that constrains the body to its plate-like configuration but performs no work in doing so. The equations of equilibrium can be expressed in weak form as

$$
\int_{\mathcal{B}} \boldsymbol{P}: \frac{\partial \stackrel{\Delta}{\boldsymbol{x}}}{\partial \boldsymbol{X}} d v=\oint_{\partial \mathcal{B}} \boldsymbol{\tau} \cdot \stackrel{\Delta}{\boldsymbol{x}} d a,
$$

where $\partial \mathcal{B}$ is the boundary of the reference configuration and $\boldsymbol{\tau}$ is the applied load vector at a point on this boundary. Hence equations of equilibrium of the constrained body have the weak form

$$
\int_{\mathcal{B}} \boldsymbol{P}_{a c t}: \frac{\partial \stackrel{\Delta}{\boldsymbol{x}}}{\partial \boldsymbol{X}} d v=\oint_{\partial \mathcal{B}} \boldsymbol{\tau} \cdot \stackrel{\Delta}{\boldsymbol{x}} d a,
$$

where $\stackrel{\Delta}{\boldsymbol{x}}$ is again a virtual displacement from a restricted class tangent to the manifold of allowed configurations.

\subsection{Cylindrical Deformations of the Axisymmetric Plate}

We consider cylindrical deformations of the plate, that is deformations of the form

$$
\boldsymbol{x}(R, \Theta, Z)=r(R) \boldsymbol{e}_{1}(\Theta)+Z \boldsymbol{k},
$$

The plate consists of a two-dimensional middle surface from which material fibers extend perpendicularly. The deformation leaves the material fibers unstretched and perpendicular to the middle surface. The only possible virtual displacements are axially symmetric perturbations of the middle surface, so in this case $\stackrel{\Delta}{\boldsymbol{x}}$ has the form

$$
\stackrel{\Delta}{\boldsymbol{x}}(R, \Theta)=\stackrel{\Delta}{r}(R) \boldsymbol{e}_{1}(\Theta),
$$


where $\stackrel{\Delta}{r}$ is any as-regular-as-needed real-valued function. The weak form of the equation of equilibrium is

$$
\int_{\text {plate }}\left(\left(\boldsymbol{e}_{1} \cdot \boldsymbol{P}_{a c t} \cdot \boldsymbol{e}_{1}\right) \stackrel{\Delta^{\prime}}{r}+\left(\boldsymbol{e}_{2} \cdot \boldsymbol{P}_{a c t} \cdot \boldsymbol{e}_{2}\right) \frac{\stackrel{\Delta}{r}}{R}\right) d v=\oint_{\partial(\text { plate })} \boldsymbol{\tau} \cdot\left(\stackrel{\Delta}{r} \boldsymbol{e}_{1}\right) d a
$$

because all other projections of the tensor-valued gradient $\partial \stackrel{\Delta}{\boldsymbol{x}} / \partial \boldsymbol{X}$ are zero.

It should be noted that

$$
\begin{aligned}
& \left(\boldsymbol{e}_{1} \cdot \boldsymbol{P}_{a c t} \cdot \boldsymbol{e}_{1}\right) \stackrel{\Delta^{\prime}}{r}+\left(\boldsymbol{e}_{2} \cdot \boldsymbol{P}_{a c t} \cdot \boldsymbol{e}_{2}\right) \frac{\stackrel{\Delta}{r}}{R} \\
& =\left(\stackrel{\Delta^{\prime}}{{ }^{\prime}} \boldsymbol{e}_{1}\right) \cdot\left(\boldsymbol{P}_{a c t} \cdot \boldsymbol{e}_{1}\right)+\left(\frac{\stackrel{r}{R}}{R} \boldsymbol{e}_{2}\right) \cdot\left(\boldsymbol{P}_{a c t} \cdot \boldsymbol{e}_{2}\right) \\
& =\left(\frac{\partial \Delta}{\partial R}\right) \cdot\left(\boldsymbol{P}_{a c t} \cdot \boldsymbol{e}_{1}\right)+\left(\frac{1}{R} \frac{\partial \Delta}{\partial \Theta}\right) \cdot\left(\boldsymbol{P}_{a c t} \cdot \boldsymbol{e}_{2}\right)+\left(\frac{\partial \Delta}{\partial Z}\right) \cdot\left(\boldsymbol{P}_{a c t} \cdot \boldsymbol{k}\right) \\
& =\frac{1}{R}\left(\frac{\partial}{\partial R} R \dot{x}\right) \cdot\left(\boldsymbol{P}_{a c t} \cdot \boldsymbol{e}_{1}\right)-\stackrel{\Delta}{\boldsymbol{x}} \cdot \boldsymbol{P}_{a c t} \cdot \boldsymbol{e}_{1}+\left(\frac{1}{R} \frac{\partial \Delta}{\partial \Theta}\right) \cdot\left(\boldsymbol{P}_{a c t} \cdot \boldsymbol{e}_{2}\right)+\left(\frac{\partial \Delta}{\partial Z}\right) \cdot\left(\boldsymbol{P}_{a c t} \cdot \boldsymbol{k}\right) \\
& =\operatorname{Div}\left(\stackrel{\Delta}{\boldsymbol{x}} \cdot \boldsymbol{P}_{a c t}\right)-\stackrel{\Delta}{\boldsymbol{x}} \cdot\left(\frac{1}{R} \frac{\partial}{\partial R}\left(R \boldsymbol{P}_{a c t} \cdot \boldsymbol{e}_{1}\right)+\frac{1}{R} \frac{\partial}{\partial \Theta}\left(\boldsymbol{P}_{a c t} \cdot \boldsymbol{e}_{2}\right)\right),
\end{aligned}
$$

where Div indicates divergence with respect to cylindrical coordinates in the reference configuration. We have assumed that since there is no extension or shear through the thickness of the plate, $\left(\boldsymbol{P}_{a c t} \cdot \boldsymbol{k}\right)$ is vertical, so that $\partial\left(\boldsymbol{P}_{a c t} \cdot \boldsymbol{k}\right) / \partial Z$ is also vertical and has zero inner product with $\stackrel{\Delta}{\boldsymbol{x}}$.

By the Divergence Theorem applied to the vector field $\stackrel{\Delta}{\boldsymbol{x}} \cdot \boldsymbol{P}_{a c t}$,

$$
\int_{\text {plate }} \operatorname{Div}\left(\stackrel{\Delta}{\boldsymbol{x}} \cdot \boldsymbol{P}_{a c t}\right) d v=\oint_{\partial(\text { plate })} \stackrel{\Delta}{\boldsymbol{x}} \cdot \boldsymbol{P}_{a c t} \cdot \boldsymbol{\nu} d a=\oint_{\partial(\text { plate })} \stackrel{\Delta}{\boldsymbol{x}} \cdot \boldsymbol{\tau} d a
$$

where $\partial$ (plate) is the boundary of the plate and $\boldsymbol{\nu}$ is the outward-pointing unit normal on this boundary. After cancellation of the surface traction terms, the weak equation of equilibrium is

$$
\int_{\text {plate }} \stackrel{\Delta}{r} \boldsymbol{e}_{1} \cdot\left(\frac{1}{R} \frac{\partial}{\partial R}\left(R \boldsymbol{P}_{a c t} \cdot \boldsymbol{e}_{1}\right)+\frac{1}{R} \frac{\partial}{\partial \Theta}\left(\boldsymbol{P}_{a c t} \cdot \boldsymbol{e}_{2}\right)\right) d v=0
$$

which must hold for all admissible $\stackrel{\Delta}{r}$.

Due to the constraints imposed on the body, we do not have an equation that determines the rate of change of stress in the $Z$-direction. However, in an induced theory such as this, we do not consider the pointwise values of stress in the three-dimensional body; rather, we consider the stress integrated across the thickness of the plate. The integrated stress is a function of the planar coordinates $R$ and $\Theta$ only. 
We take the weak equation of equilibrium and write the integral over the plate as an iterated integral, first in $Z$ and then in the planar coordinates:

$$
\int_{\text {slice }} \stackrel{\Delta}{r} \boldsymbol{e}_{1} \cdot\left[\int_{H_{1}}^{H_{2}}\left(\frac{1}{R} \frac{\partial}{\partial R}\left(R \boldsymbol{P}_{a c t} \cdot \boldsymbol{e}_{1}\right)+\frac{1}{R} \frac{\partial}{\partial \Theta}\left(\boldsymbol{P}_{a c t} \cdot \boldsymbol{e}_{2}\right)\right) d Z\right] R d R d \Theta=0
$$

We change the order of differentiation and $Z$-integration:

$$
\int_{\text {slice }} \stackrel{\Delta}{r} \boldsymbol{e}_{1} \cdot\left(\frac{1}{R} \frac{\partial}{\partial R} \int_{H_{1}}^{H_{2}}\left(R \boldsymbol{P}_{a c t} \cdot \boldsymbol{e}_{1}\right) d Z+\frac{1}{R} \frac{\partial}{\partial \Theta} \int_{H_{1}}^{H_{2}}\left(\boldsymbol{P}_{a c t} \cdot \boldsymbol{e}_{2}\right) d Z\right) R d R d \Theta=0
$$

By our assumptions of symmetry, $\boldsymbol{P}_{a c t} \cdot \boldsymbol{e}_{2}$ must be proportional to $\boldsymbol{e}_{2}$, so $\boldsymbol{P}_{a c t} \cdot \boldsymbol{e}_{2}=\left(\boldsymbol{e}_{2} \cdot \boldsymbol{P}_{a c t} \cdot \boldsymbol{e}_{2}\right) \boldsymbol{e}_{2}$. And by symmetry, the coefficient $\left(\boldsymbol{e}_{2} \cdot \boldsymbol{P}_{\text {act }} \cdot \boldsymbol{e}_{2}\right)$ is $\Theta$-independent, so

$$
\begin{aligned}
\frac{\partial}{\partial \Theta} \int_{H_{1}}^{H_{2}}\left(\boldsymbol{P}_{a c t} \cdot \boldsymbol{e}_{2}\right) d Z & =\frac{\partial}{\partial \Theta} \int_{H_{1}}^{H_{2}}\left(\boldsymbol{e}_{2} \cdot \boldsymbol{P}_{a c t} \cdot \boldsymbol{e}_{2}\right) \boldsymbol{e}_{2} d Z \\
& =\frac{\partial \boldsymbol{e}_{2}}{\partial \Theta} \int_{H_{1}}^{H_{2}}\left(\boldsymbol{e}_{2} \cdot \boldsymbol{P}_{a c t} \cdot \boldsymbol{e}_{2}\right) d Z \\
& =-\boldsymbol{e}_{1} \int_{H_{1}}^{H_{2}}\left(\boldsymbol{e}_{2} \cdot \boldsymbol{P}_{a c t} \cdot \boldsymbol{e}_{2}\right) d Z
\end{aligned}
$$

Our weak equation of equilibrium is now

$$
\int_{\text {slice }} \stackrel{\Delta}{r}\left(\frac{\partial}{\partial R} R \int_{H_{1}}^{H_{2}}\left(\boldsymbol{e}_{1} \cdot \boldsymbol{P}_{a c t} \cdot \boldsymbol{e}_{1}\right) d Z-\int_{H_{1}}^{H_{2}}\left(\boldsymbol{e}_{2} \cdot \boldsymbol{P}_{a c t} \cdot \boldsymbol{e}_{2}\right) d Z\right) d R d \Theta=0 .
$$

Our symmetry assumption implies that the remaining mechanical quantities are $\Theta$-independent, so we can integrate over $\Theta$ and view the functions in the result as functions of $R$ alone:

$$
2 \pi \int_{\text {slice }} \stackrel{\Delta}{r}\left(\frac{d}{d R} R \int_{H_{1}}^{H_{2}}\left(\boldsymbol{e}_{1} \cdot \boldsymbol{P}_{a c t} \cdot \boldsymbol{e}_{1}\right) d Z-\int_{H_{1}}^{H_{2}}\left(\boldsymbol{e}_{2} \cdot \boldsymbol{P}_{a c t} \cdot \boldsymbol{e}_{2}\right) d Z\right) d R=0 .
$$

If this is to hold for all admissible $\stackrel{\Delta}{r}$, then the other factor of the $R$-integrand must be identically zero:

$$
\frac{d}{d R}\left(R \int_{H_{1}}^{H_{2}}\left(\boldsymbol{e}_{1} \cdot \boldsymbol{P}_{a c t} \cdot \boldsymbol{e}_{1}\right) d Z\right)-\int_{H_{1}}^{H_{2}}\left(\boldsymbol{e}_{2} \cdot \boldsymbol{P}_{a c t} \cdot \boldsymbol{e}_{2}\right) d Z=0
$$

This is the equation of equilibrium for our constrained axisymmetric plate. Closure of the system of differential equations requires a constitutive relation.

\section{Neo-Hookean Constitutive Relation}

We consider a compressible neo-Hookean constitutive relation with strain energy density

$$
W\left(\lambda_{1}, \lambda_{2}, \lambda_{3}\right)=\frac{\mu}{2}\left(\lambda_{1}^{2}+\lambda_{2}^{2}+\lambda_{3}^{2}-3-2 \ln J\right)+\lambda(J-1-\ln J) ;
$$

this is very similar to the strain-energy found in Problem 6.3.1 of [21]. Here $\mu$ and $\lambda$ can be identified, for small deformations, with the Lamé parameters of linear elasticity, $\lambda_{1}^{2}, \lambda_{2}^{2}$, and 
$\lambda_{3}^{2}$ are the eigenvalues of the left Cauchy-Green tensor $\boldsymbol{F} \cdot \boldsymbol{F}^{T}$, and $J=\lambda_{1} \lambda_{2} \lambda_{3}=\operatorname{det} \boldsymbol{F}$. The principal components of the active Cauchy stress (the constitutively-defined portion of the Cauchy stress) are then

$$
\left(\boldsymbol{T}_{a c t}\right)_{i}=\frac{\lambda_{i}}{J} \frac{\partial W}{\partial \lambda_{i}}=\frac{1}{J}\left(\mu\left(\lambda_{i}^{2}-1\right)+\lambda(J-1)\right) ;
$$

see section 4.3 of [21]. The full active Cauchy stress tensor is

$$
\boldsymbol{T}_{a c t}=\frac{1}{J}\left(\mu\left(\lambda_{1}^{2} \boldsymbol{e}_{1} \otimes \boldsymbol{e}_{1}+\lambda_{2}^{2} \boldsymbol{e}_{2} \otimes \boldsymbol{e}_{2}+\lambda_{3}^{2} \boldsymbol{k} \otimes \boldsymbol{k}\right)+(\lambda J-(\lambda+\mu)) \boldsymbol{I}\right) .
$$

If a deformation gradient is decomposed as $\boldsymbol{F}=\boldsymbol{A} \cdot \boldsymbol{G}$, where $\boldsymbol{G}$ corresponds to non-elastic growth, then [8] demonstrates that the appropriate constitutive definition of the Cauchy stress tensor is to use the same formula as in growthless hyperelasticity, with the $\operatorname{argument} \boldsymbol{F} \cdot \boldsymbol{F}^{T}$ replaced by $\boldsymbol{A} \cdot \boldsymbol{A}^{T}$. Hence we use Eq. (38) as the definition of $\boldsymbol{T}_{\text {act }}$, only now each $\lambda_{i}^{2}$ is an eigenvalue of $\boldsymbol{A} \cdot \boldsymbol{A}^{T}$ and $J=\operatorname{det} \boldsymbol{A}$.

The Cauchy stress is an Eulerian tensor, while the differential equations have been derived in terms of the active portion $\boldsymbol{P}_{a c t}$ of the first Piola-Kirchhoff stress, which is a two-point tensor that maps normal vectors in the reference configuration to stress vectors in the current (post-growth, post-elastic response) configuration. The full deformation gradient $\boldsymbol{F}$ is required to convert Eulerian and Lagrangian tensors into two-point tensors, so the relation between $\boldsymbol{T}_{\text {act }}$ and $\boldsymbol{P}_{\text {act }}$ has the same form as in traditional elasticity:

$$
\boldsymbol{P}_{a c t}=(\operatorname{det} \boldsymbol{F}) \boldsymbol{T}_{a c t} \cdot \boldsymbol{F}^{-T} .
$$

For our particular problem we do not need to compute the full first Piola-Kirchhoff stress tensor since we need only the projections $\boldsymbol{e}_{1} \cdot \boldsymbol{P}_{\text {act }} \cdot \boldsymbol{e}_{1}$ and $\boldsymbol{e}_{2} \cdot \boldsymbol{P}_{\text {act }} \cdot \boldsymbol{e}_{2}$.

$$
\begin{aligned}
\boldsymbol{e}_{1} \cdot \boldsymbol{P}_{a c t} \cdot \boldsymbol{e}_{1} & =(\operatorname{det} \boldsymbol{F}) \boldsymbol{e}_{1} \cdot \boldsymbol{T}_{a c t} \cdot \boldsymbol{F}^{-T} \cdot \boldsymbol{e}_{1} \\
& =\frac{(\operatorname{det} \boldsymbol{A})(\operatorname{det} \boldsymbol{G})}{J}\left(\mu \lambda_{1}^{2}+\lambda J-(\lambda+\mu)\right) \boldsymbol{e}_{1} \cdot\left(\frac{1}{r^{\prime}} \boldsymbol{e}_{1}\right) \\
& =(\operatorname{det} \boldsymbol{G})\left(\mu\left(\frac{r^{\prime}}{\gamma_{1}}\right)^{2}+\lambda J-(\lambda+\mu)\right) \frac{1}{r^{\prime}} \\
& =\gamma_{1} \gamma_{2}\left(\mu \frac{r^{\prime}}{\gamma_{1}^{2}}+\lambda \frac{r / R}{\gamma_{1} \gamma_{2}}-\frac{\lambda+\mu}{r^{\prime}}\right) \\
& =\frac{\mu \gamma_{2}}{\gamma_{1}} r^{\prime}+\lambda \frac{r}{R}-\frac{(\lambda+\mu) \gamma_{1} \gamma_{2}}{r^{\prime}} .
\end{aligned}
$$

A similar calculation shows that

$$
\boldsymbol{e}_{2} \cdot \boldsymbol{P}_{a c t} \cdot \boldsymbol{e}_{2}=\frac{\mu \gamma_{1}}{\gamma_{2}} \frac{r}{R}+\lambda r^{\prime}-\frac{(\lambda+\mu) \gamma_{1} \gamma_{2}}{r / R}
$$

The flatness of the middle surface and the lack of stretching or shear through the thickness have ensured a complete lack of $Z$-dependence, so the integrated stresses are

$$
\begin{aligned}
\int_{H_{1}}^{H_{2}}\left(\boldsymbol{e}_{1} \cdot \boldsymbol{P}_{a c t} \cdot \boldsymbol{e}_{1}\right) d Z & =\left(H_{2}-H_{1}\right)\left(\frac{\mu \gamma_{2}}{\gamma_{1}} r^{\prime}+\lambda \frac{r}{R}-\frac{(\lambda+\mu) \gamma_{1} \gamma_{2}}{r^{\prime}}\right) \\
\int_{H_{1}}^{H_{2}}\left(\boldsymbol{e}_{2} \cdot \boldsymbol{P}_{a c t} \cdot \boldsymbol{e}_{2}\right) d Z & =\left(H_{2}-H_{1}\right)\left(\frac{\mu \gamma_{1}}{\gamma_{2}} \frac{r}{R}+\lambda r^{\prime}-\frac{(\lambda+\mu) \gamma_{1} \gamma_{2}}{r / R}\right) .
\end{aligned}
$$


This leaves us the ordinary differential equation

$$
\frac{d}{d R}\left(R\left(\frac{\mu \gamma_{2}}{\gamma_{1}} r^{\prime}+\lambda \frac{r}{R}-\frac{(\lambda+\mu) \gamma_{1} \gamma_{2}}{r^{\prime}}\right)\right)=\frac{\mu \gamma_{1}}{\gamma_{2}} \frac{r}{R}+\lambda r^{\prime}-\frac{(\lambda+\mu) \gamma_{1} \gamma_{2}}{r / R}
$$

After differentiation and cancellation of $\lambda r^{\prime}$ from each side, we have

$$
\begin{aligned}
& \left(\frac{\mu \gamma_{2}}{\gamma_{1}}\right)^{\prime} R r^{\prime}+\frac{\mu \gamma_{2}}{\gamma_{1}} r^{\prime}+\frac{\mu \gamma_{2}}{\gamma_{1}} R r^{\prime \prime}-\frac{(\lambda+\mu) \gamma_{1} \gamma_{2}}{r^{\prime}}-\frac{(\lambda+\mu) R\left(\gamma_{1} \gamma_{2}\right)^{\prime}}{r^{\prime}}+\frac{(\lambda+\mu) R \gamma_{1} \gamma_{2}}{\left(r^{\prime}\right)^{2}} r^{\prime \prime} \\
= & \frac{\mu \gamma_{1}}{\gamma_{2}} \frac{r}{R}-\frac{(\lambda+\mu) R \gamma_{1} \gamma_{2}}{r / R} .
\end{aligned}
$$

We assume that $r$ is at least twice continuously differentiable and that $r^{\prime}$ is bounded away from zero. By the boundedness of derivatives near $R=0$ and the fact that $r(R) / R \rightarrow r^{\prime}(0)$ as $R \rightarrow 0$, the limiting form of the equation above is, after cancellation,

$$
\frac{\gamma_{2}(0)}{\gamma_{1}(0)} r^{\prime}(0)=\frac{\gamma_{1}(0)}{\gamma_{2}(0)} r^{\prime}(0)
$$

The conclusion is that we need either $r^{\prime}(0)=0$ or $\gamma_{1}(0)=\gamma_{2}(0)$ for a configuration without a cavity.

We consider the first possibility. Suppose that near $R=0, r(R)=A(R) R^{\alpha}$, where $A$ is a slowly-varying function bounded above zero near $R=0$. Since we want $r$ to be twice continuously differentiable, we assume $\alpha=1$ or $\alpha \geq 2$. If $\alpha=1$, then $r^{\prime}(0) \neq 0$, and we assume $\gamma_{1}(0)=\gamma_{2}(0)$. If $\alpha \geq 2$, then

$$
r^{\prime}=\alpha A R^{\alpha-1}+A^{\prime} R^{\alpha}, \quad r^{\prime \prime}=(\alpha-1) \alpha A R^{\alpha-2}+2 \alpha A^{\prime} R^{\alpha-1}+A^{\prime \prime} R^{\alpha},
$$

and the most singular terms in Eq. (45) are

$$
\begin{aligned}
-\frac{(\lambda+\mu)\left(\gamma_{1} \gamma_{2}\right)^{\prime}}{r^{\prime} / R} & \approx-\left.\frac{(\lambda+\mu)\left(\gamma_{1} \gamma_{2}\right)^{\prime}}{\alpha A}\right|_{R=0} R^{2-\alpha} \\
-\frac{(\lambda+\mu) \gamma_{1} \gamma_{2}}{r / R^{2}} & \approx-\left.\frac{(\lambda+\mu) \gamma_{1} \gamma_{2}}{A}\right|_{R=0} R^{2-\alpha} .
\end{aligned}
$$

Requiring that the coefficients agree at $R=0$ would put undue restrictions on the types of growth functions $\gamma_{i}$ we may consider. We opt for the less restrictive alternative: $\gamma_{1}(0)=\gamma_{2}(0)$.

\section{Conversion to a Dynamical System}

We perform the change of variables described in section X.3 of [3]. Let $n$ be the radial stress,

$$
n=\frac{\mu \gamma_{2}}{\gamma_{1}} r^{\prime}+\lambda \frac{r}{R}-\frac{(\lambda+\mu) \gamma_{1} \gamma_{2}}{r^{\prime}}
$$

and let

$$
\tau=\frac{r}{R}
$$


Finding $r^{\prime}$ as a function of $n$ yields two solutions, only of which is positive. The flatness of the middle surface requires that $r^{\prime}$ be positive, so we choose the positive solution:

$r^{\prime}=\frac{\gamma_{1}}{2 \mu \gamma_{2}}\left(n-\lambda \frac{r}{R}+\sqrt{\left(n-\lambda \frac{r}{R}\right)^{2}+4 \mu(\lambda+\mu) \gamma_{2}^{2}}\right)=\frac{\gamma_{1}}{2 \mu \gamma_{2}}\left(n-\lambda \tau+\sqrt{(n-\lambda \tau)^{2}+4 \mu(\lambda+\mu) \gamma_{2}^{2}}\right)$

The differential equation can now be stated in terms of $n$ and $\tau$ :

$$
\begin{aligned}
\frac{d}{d R}(R n) & =\frac{\mu \gamma_{1}}{\gamma_{2}} \frac{r}{R}+\lambda r^{\prime}-\frac{(\lambda+\mu) \gamma_{1} \gamma_{2}}{r / R} \\
& =\frac{\mu \gamma_{1}}{\gamma_{2}} \tau+\underbrace{\frac{\lambda \gamma_{1}}{2 \mu \gamma_{2}}\left(n-\lambda \tau+\sqrt{(n-\lambda \tau)^{2}+4 \mu(\lambda+\mu) \gamma_{2}^{2}}\right)}_{\lambda r^{\prime}}-\frac{(\lambda+\mu) \gamma_{1} \gamma_{2}}{\tau} .
\end{aligned}
$$

Note also that

$$
\frac{d}{d R}(R \tau)=r^{\prime}=\frac{\gamma_{1}}{2 \mu \gamma_{2}}\left(n-\lambda \tau+\sqrt{(n-\lambda \tau)^{2}+4 \mu(\lambda+\mu) \gamma_{2}^{2}}\right)
$$

We define a new independent variable $s$ by

$$
R=R_{\max } e^{s-1}, \quad \text { or } \quad s=\ln \left(\frac{R}{R_{\max }}\right)+1 .
$$

If we set $\widetilde{n}(s)=n(R)$ and $\widetilde{\tau}(s)=\tau(R)$, then the $R$ - and $s$-derivatives are related by

$$
\begin{aligned}
& \frac{d}{d R}(R n)=\frac{d}{d R}(R \widetilde{n})=\widetilde{n}+R \frac{d s}{d R} \frac{d \widetilde{n}}{d s}=\widetilde{n}+\frac{d \widetilde{n}}{d s} \\
& \frac{d}{d R}(R \tau)=\frac{d}{d R}(R \widetilde{\tau})=\widetilde{\tau}+R \frac{d s}{d R} \frac{d \widetilde{\tau}}{d s}=\widetilde{\tau}+\frac{d \widetilde{\tau}}{d s}
\end{aligned}
$$

where the prime on $\widetilde{n}$ and $\widetilde{\tau}$ indicates differentiation with respect to $s$. The differential equations in $\widetilde{n}$ and $\widetilde{\tau}$ are

$$
\begin{aligned}
& \frac{d \widetilde{n}}{d s}=\frac{\mu \widetilde{\gamma}_{1}}{\widetilde{\gamma}_{2}} \widetilde{\tau}+\frac{\lambda \widetilde{\gamma}_{1}}{2 \mu \widetilde{\gamma}_{2}}\left(\widetilde{n}-\lambda \widetilde{\tau}+\sqrt{(\widetilde{n}-\lambda \widetilde{\tau})^{2}+4 \mu(\lambda+\mu) \widetilde{\gamma}_{2}^{2}}\right)-\frac{(\lambda+\mu) \widetilde{\gamma}_{1} \widetilde{\gamma}_{2}}{\widetilde{\tau}}-\widetilde{n} \\
& \frac{d \widetilde{\tau}}{d s}=\frac{\widetilde{\gamma}_{1}}{2 \mu \widetilde{\gamma}_{2}}\left(\widetilde{n}-\lambda \widetilde{\tau}+\sqrt{(\widetilde{n}-\lambda \widetilde{\tau})^{2}+4 \mu(\lambda+\mu) \widetilde{\gamma}_{2}^{2}}\right)-\widetilde{\tau}
\end{aligned}
$$

If we set $N=\widetilde{n} / \mu$ and $\kappa=\lambda / \mu$, then we can simplify these equations to:

$$
\begin{aligned}
\frac{d N}{d s} & =\frac{\widetilde{\gamma}_{1}}{\widetilde{\gamma}_{2}} \widetilde{\kappa}+\frac{\kappa \widetilde{\gamma}_{1}}{2 \widetilde{\gamma}_{2}}\left(N-\kappa \widetilde{\tau}+\sqrt{(N-\kappa \widetilde{\tau})^{2}+4(1+\kappa) \widetilde{\gamma}_{2}^{2}}\right)-\frac{(1+\kappa) \widetilde{\gamma}_{1} \widetilde{\gamma}_{2}}{\widetilde{\tau}}-N \\
\frac{d \widetilde{\tau}}{d s} & =\frac{\widetilde{\gamma}_{1}}{2 \widetilde{\gamma}_{2}}\left(N-\kappa \widetilde{\tau}+\sqrt{(N-\kappa \widetilde{\tau})^{2}+4(1+\kappa) \widetilde{\gamma}_{2}^{2}}\right)-\widetilde{\tau}
\end{aligned}
$$

where $\widetilde{\gamma}_{i}(s)=\gamma_{i}(R)$. 
Were it not for the $s$-dependence of the $\widetilde{\gamma}_{i}$, these equations would be autonomous and could be subjected to a fairly straight-forward phase-plane analysis. If the $\widetilde{\gamma}_{i}$ satisfied some autonomous differential equations, then adding those equations to the pair above would form an autonomous system. Below we consider several examples of $\widetilde{\gamma}_{i}$ that satisfy autonomous equations.

Finally, we add the following two boundary conditions to our system of differential equations:

$$
\begin{aligned}
N(1) & =0, & & \left(\text { zero radial stress at } R=R_{\max }\right) \\
\lim _{s \rightarrow-\infty} R_{\max } e^{s-1} \widetilde{\tau}(s) & =0 . & & (r(0)=0)
\end{aligned}
$$

\section{No Radial Growth: $\gamma_{1}(R) \equiv 1$}

Suppose that $\gamma_{1}(R) \equiv 1$ and $\gamma_{2}(R)=a+b\left(R / R_{\max }\right)^{2}$. Since we want $\gamma_{1}(0)=\gamma_{2}(0)$, we set $a=1$. Then $\gamma_{2}$ satisfies

$$
\frac{d}{d R}\left(R \gamma_{2}(R)\right)=\frac{d}{d R}\left(R+\frac{b}{R_{\max }^{2}} R^{3}\right)=1+3 b\left(\frac{R}{R_{\max }}\right)^{2}=1+3\left(\gamma_{2}(R)-1\right)
$$

so $\widetilde{\gamma}_{2}$ satisfies

$$
\frac{\widetilde{\gamma}_{2}}{d s}+\widetilde{\gamma}_{2}=1+3\left(\widetilde{\gamma}_{2}-1\right), \quad \text { or } \quad \frac{\widetilde{\gamma}_{2}}{d s}=2\left(\widetilde{\gamma}_{2}-1\right)
$$

We have the system of autonomous equations or dynamical system

$$
\begin{aligned}
\frac{d \widetilde{\tau}}{d s} & =\frac{1}{2 \widetilde{\gamma}_{2}}\left(N-\kappa \widetilde{\tau}+\sqrt{(N-\kappa \widetilde{\tau})^{2}+4(1+\kappa) \widetilde{\gamma}_{2}^{2}}\right)-\widetilde{\tau} \\
\frac{d N}{d s} & =\frac{\widetilde{\tau}}{\widetilde{\gamma}_{2}}+\frac{\kappa}{2 \widetilde{\gamma}_{2}}\left(N-\kappa \widetilde{\tau}+\sqrt{(N-\kappa \widetilde{\tau})^{2}+4(1+\kappa) \widetilde{\gamma}_{2}^{2}}\right)-\frac{(1+\kappa) \widetilde{\gamma}_{2}}{\widetilde{\tau}}-N \\
\frac{d \widetilde{\gamma}_{2}}{d s} & =2\left(\widetilde{\gamma}_{2}-1\right) .
\end{aligned}
$$

We will often summarize this system of differential equations by

$$
\frac{d \mathbf{y}}{d s}=\mathbf{f}(\mathbf{y})
$$

where $\mathbf{y}=\left(\widetilde{\tau}, N, \widetilde{\gamma}_{2}\right)^{T}$. Points $\mathbf{y} \in \mathbb{R}^{3}$ will be described via $\widetilde{\tau}-, N$-, and $\widetilde{\gamma}_{2}$-coordinates.

Definition: Domain The domain of $\mathbf{f}$ is

$$
\mathcal{D}(\mathbf{f})=\left\{\left(\widetilde{\tau}, N, \widetilde{\gamma}_{2}\right) \in \mathbb{R}^{3}: \mathbf{f}\left(\widetilde{\tau}, N, \widetilde{\gamma}_{2}\right) \text { is defined }\right\}
$$

In the case at hand, $\mathcal{D}(\mathbf{f})$ consists of all of $\mathbb{R}^{3}$ except for the planes $\widetilde{\tau}=0$ and $\widetilde{\gamma}_{2}=0$.

Definition: Maximal Interval of Existence Each point $\mathbf{y}_{0} \in \mathcal{D}(\mathbf{f})$ can be considered as the initial condition of some solution of Eqs. (66)-(68):

$$
\frac{d \mathbf{y}}{d s}=\mathbf{f}(\mathbf{y}), \quad \mathbf{y}(0)=\mathbf{y}_{0}
$$


The maximal interval of existence $I\left(\mathbf{y}_{0}\right)$ is the largest interval of $s$-values (both positive and negative) for which the solution of Eq. (71) exists and is unique.

Theorem: Uniqueness If $\mathbf{f}$ and the $\widetilde{\tau}-, N$-, and $\widetilde{\gamma}_{2}$-derivatives of $\mathbf{f}$ are continuous on the "box" $\left\{\mathbf{y} \in \mathbb{R}^{3}:\left\|\mathbf{y}-\mathbf{y}_{0}\right\|<b\right\}$, then Eq. (71) has at most one solution.

For proof, see e.g. Section 3.3 of [5]. Note that for Eqs. (66)-(68), f is continuously differentiable in any finite box bounded away from $\widetilde{\tau}=0$ and $\widetilde{\gamma}_{2}=0$.

Definition: Orbit If $s \mapsto \mathbf{y}(s)$ is a solution of Eq. (71), then the curve

$$
\left\{\mathbf{y}(s) \in \mathbb{R}^{3}: s \in I\left(\mathbf{y}_{0}\right)\right\}
$$

is called an orbit or trajectory of Eq. (71). We will often say that the solution $s \mapsto \mathbf{y}(s)$ traces out its orbit.

One of the most important properties of orbits of autonomous systems of differential equations is that in regions of $\mathcal{D}(\mathbf{f})$ where $\mathbf{f}$ is continuously differentiable, distinct orbits do not intersect. Hence, if $s \mapsto \mathbf{y}(s)$ and $s \mapsto \mathbf{z}(s)$ are solutions of Eq. (71) with different initial conditions and $\mathbf{y}\left(s_{1}\right)=\mathbf{z}\left(s_{2}\right)$ for some $s_{1} \neq s_{1}$, then $\mathbf{z}(s)=\mathbf{y}\left(s+s_{1}-s_{2}\right)$, i.e. $s \mapsto \mathbf{z}(s)$ is essentially $s \mapsto \mathbf{y}(s)$ with a constant shift or argument.

Definition: Projection Much of the analysis will focus on the coordinates $(\widetilde{\tau}, N)$. If $s \mapsto\left(\widetilde{\tau}(s), N(s), \widetilde{\gamma}_{2}(s)\right)$ is a solution of Eqs. (66)-(68), then we will refer to the function $s \mapsto(\widetilde{\tau}(s), N(s))$ as the solution's projection into the $(\widetilde{\tau}, N)$-plane. We will also refer to the set of points

$$
\{(\widetilde{\tau}(s), N(s)): s \in \text { maximal interval of existence }\},
$$

as the projection of the orbit.

\subsection{Fixed Points and Linearization}

The fixed points of Eqs. (66)-(68) form curves whose coordinates satisfy

$$
\widetilde{\gamma}_{2}=1, \quad N=(1+\kappa)\left(\widetilde{\tau}+\widetilde{\tau}^{-1}\right) .
$$

The coordinates of the fixed points with $\widetilde{\tau}>0$ satisfy

$$
\widetilde{\gamma}_{2}=1, \quad \widetilde{\tau}=\frac{N+\sqrt{N^{2}+4(1+\kappa)^{2}}}{2(1+\kappa)} .
$$

At each fixed point, the derivative of the right-hand sides of Eqs. (66)-(68) has the form

$$
\begin{aligned}
& D \mathbf{f}\left(\widetilde{\tau},(1+\kappa)\left(\widetilde{\tau}+\tilde{\tau}^{-1}\right), 1\right) \\
= & \left(\begin{array}{ccc}
-\frac{(1+\kappa)\left(1+\tilde{\tau}^{2}\right)}{1+\kappa+\tilde{\tau}^{2}} & \frac{\tilde{\tau}^{2}}{1+\kappa+\tilde{\tau}^{2}} & \frac{\tilde{\tau}\left(1+\kappa-\tilde{\tau}^{2}\right)}{1+\kappa+\tilde{\tau}^{2}} \\
\frac{(1+\kappa)\left(1+\tilde{\tau}^{2}\right)\left(1+\kappa-(-1+\kappa) \tilde{\tau}^{2}\right)}{\tilde{\tau}^{2}\left(1+\kappa+\tilde{\tau}^{2}\right)} & \frac{-1-\kappa+(-1+\kappa) \tilde{\tau}^{2}}{1+\kappa+\tilde{\tau}^{2}} & -\frac{1+\kappa}{\tilde{\tau}}-\tilde{\tau}-\kappa \tilde{\tau}+\frac{2 \kappa(1+\kappa) \tilde{\tau}}{1+\kappa+\tilde{\tau}^{2}} \\
0 & 0 & 2
\end{array}\right) .
\end{aligned}
$$


This matrix has eigenvalues $-2,0$, and 2, regardless of the values of $\kappa$ and $\widetilde{\tau}$. This indicates the presence of a stable manifold, a center manifold, and an unstable manifold attached to each fixed point; see [22].

Each of these manifolds is traced out by a solution of Eqs. (66)-(68). The unstable manifold is "unstable" in the sense that the solution that traces it out approaches the fixed point at an exponential rate as $s \rightarrow-\infty$, i.e. as the dynamical system is run backwards. The eigenvector corresponding to the eigenvalue 2 describes the direction from which this solution approaches the fixed point as $s \rightarrow-\infty$.

For a solution corresponding to a configuration of the plate, $\widetilde{\gamma}_{2}(s)$ is non-constant and $\widetilde{\gamma}_{2}(s) \downarrow 1$ as $s \rightarrow-\infty$. The only eigenvector of the derivative matrix that has nonzero $\widetilde{\gamma}_{2^{-}}$ component is the one associated with the eigenvalue 2 :

$$
\left(-\frac{-\tilde{\tau}-\kappa \tilde{\tau}+2 \tilde{\tau}^{3}}{4\left(1+\kappa+\tilde{\tau}^{2}\right)},-\frac{1+2 \kappa+\kappa^{2}+3 \tilde{\tau}^{2}+2 \kappa \tilde{\tau}^{2}-\kappa^{2} \tilde{\tau}^{2}+2 \tilde{\tau}^{4}+2 \kappa \tilde{\tau}^{4}}{4 \tilde{\tau}\left(1+\kappa+\tilde{\tau}^{2}\right)}, 1\right)^{T} .
$$

This means that the only orbit attached to a fixed point that could possibly correspond to a configuration of the body is an unstable manifold. We will show in the next section that there is an unstable manifold that corresponds to a plate with zero radial stress at its periphery.

\subsection{Existence of Solution}

We will demonstrate that there is one solution that traces out an unstable manifold and whose $N$-coordinate satisfies $N(1)=0$.

For each $s>-\infty$, we define the horizontal isocline

$$
\Gamma_{h}(s)=\left\{(\widetilde{\tau}, N): \widetilde{\tau}=\frac{N+\sqrt{N^{2}+4(1+\kappa)\left(\kappa+\widetilde{\gamma}_{2}(s)\right) \widetilde{\gamma}_{2}(s)}}{2\left(\kappa+\widetilde{\gamma}_{2}(s)\right)}\right\}
$$

and the vertical isocline

$$
\Gamma_{v}(s)=\left\{(\widetilde{\tau}, N): \widetilde{\tau}>0 \text { and } \frac{1}{2 \widetilde{\gamma}_{2}(s)}\left(N-\kappa \widetilde{\tau}+\sqrt{(N-\kappa \widetilde{\tau})^{2}+4(1+\kappa) \widetilde{\gamma}_{2}(s)^{2}}\right)-\widetilde{\tau}=0\right\} .
$$

$\Gamma_{h}(s)$ consists of the points in the $(\widetilde{\tau}, N)$-plane with $\widetilde{\tau}>0$ where the vector field has zero

$\widetilde{\tau}$-component at $s . \Gamma_{v}(s)$ consists of the points in the same half-plane where the vector field has zero $N$-component at $s$. If $\kappa>1$, then $\Gamma_{v}(s)$ has two branches, one above $\Gamma_{h}(s)$ and one below. See Figure 4.

Claim 1 At $s=-\infty, \Gamma_{h}(s)$ and $\Gamma_{v}(s)$ coincide with $\Gamma(-\infty)$.

Proof of Claim 1 In the defining equation of the horizontal isocline $\Gamma_{h}(s)$, set $\widetilde{\gamma}_{2}=1$. This coincides with the equation for the curve of fixed points. Plug this formula for $\widetilde{\tau}$ into the defining equation of the vertical isocline (with $\widetilde{\gamma}_{2}$ set to 1 ) and the equation becomes a tautology. 


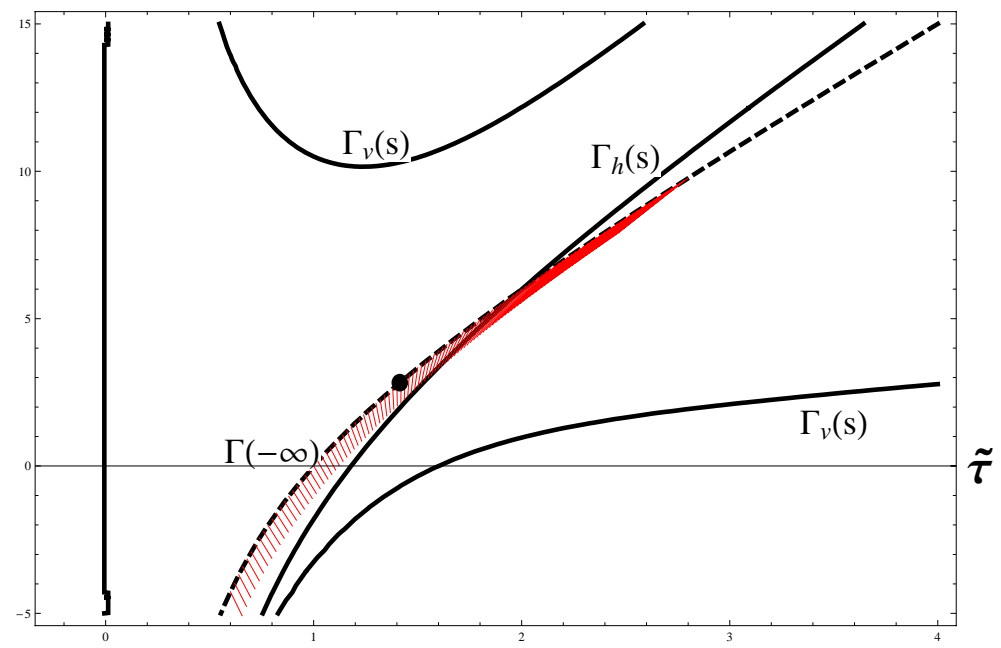

Figure 4: Isoclines in the $(\widetilde{\tau}, N)$-plane at some $s>-\infty$. The presence of the upper branch of $\Gamma_{v}(s)$ indicates that $\kappa>1$ in this example. The line segments attached to $\Gamma(-\infty)$ show the directions of the unstable eigenvectors.

Claim 2 At $s=1$, the projection of each solution that traces out an unstable manifold lies in the region between $\Gamma(-\infty)$ and the lower branch of $\Gamma_{v}(1)$.

Proof of Claim 2 First we note that the $N$-component of the "unstable eigenvector" in Eq. (77),

$$
-\frac{(1+\kappa)\left(2 \tilde{\tau}^{4}+\left(3-\kappa^{2}\right) \tilde{\tau}^{2}+(1+\kappa)\right)}{4 \tilde{\tau}\left(1+\kappa+\tilde{\tau}^{2}\right)}
$$

is negative for all real $\widetilde{\tau}$. Hence each fixed point is asymptotically approached from below (as $s \rightarrow-\infty)$, which means each fixed point is approached asymptotically from the region between $\Gamma(-\infty)$ and the lower branch of $\Gamma_{v}(s)$.

Figure 5 shows the vector field for the dynamical system run in reverse. If $(\widetilde{\tau}(s), N(s))$ is to the left of $\Gamma(-\infty)$ for some $s>-\infty$, then $\widetilde{\tau}(s) \rightarrow 0$ as $s \rightarrow-\infty$, and the orbit does not approach a fixed point. If $(\widetilde{\tau}(s), N(s))$ is to the right of thew lower branch of $\Gamma_{v}(s)$ for some $s>-\infty$, then $\widetilde{\tau}(s) \rightarrow \infty$ as $s \rightarrow-\infty$, and the orbit does not approach a fixed point.

In particular, each solution whose orbit is an unstable manifold, lies in the region between $\Gamma(-\infty)$ and the lower branch of $\Gamma_{v}(1)$ at $s=1$.

Definition: Flow If we view each point $\mathbf{y}_{0} \in \mathcal{D}(\mathbf{f})$ as the initial condition of the problem

$$
\frac{d \mathbf{y}}{d s}=\mathbf{f}(\mathbf{y}), \quad \mathbf{y}(0)=\mathbf{y}_{0},
$$

then the flow of that dynamical system is a function defined as

$$
\Phi\left(\mathbf{y}_{0}, s\right)=\mathbf{y}(s), \quad s \in I\left(\mathbf{y}_{0}\right) .
$$

Let $\mathcal{E}$ be an open subset of $\mathcal{D}(\mathbf{f})$ such that $\mathbf{f} \in C^{1}(\mathcal{E})$. Define

$$
\Omega=\left\{\left(s, \mathbf{y}_{0}\right) \in \mathbb{R} \times \mathcal{E}: s \in I\left(\mathbf{y}_{0}\right)\right\} .
$$




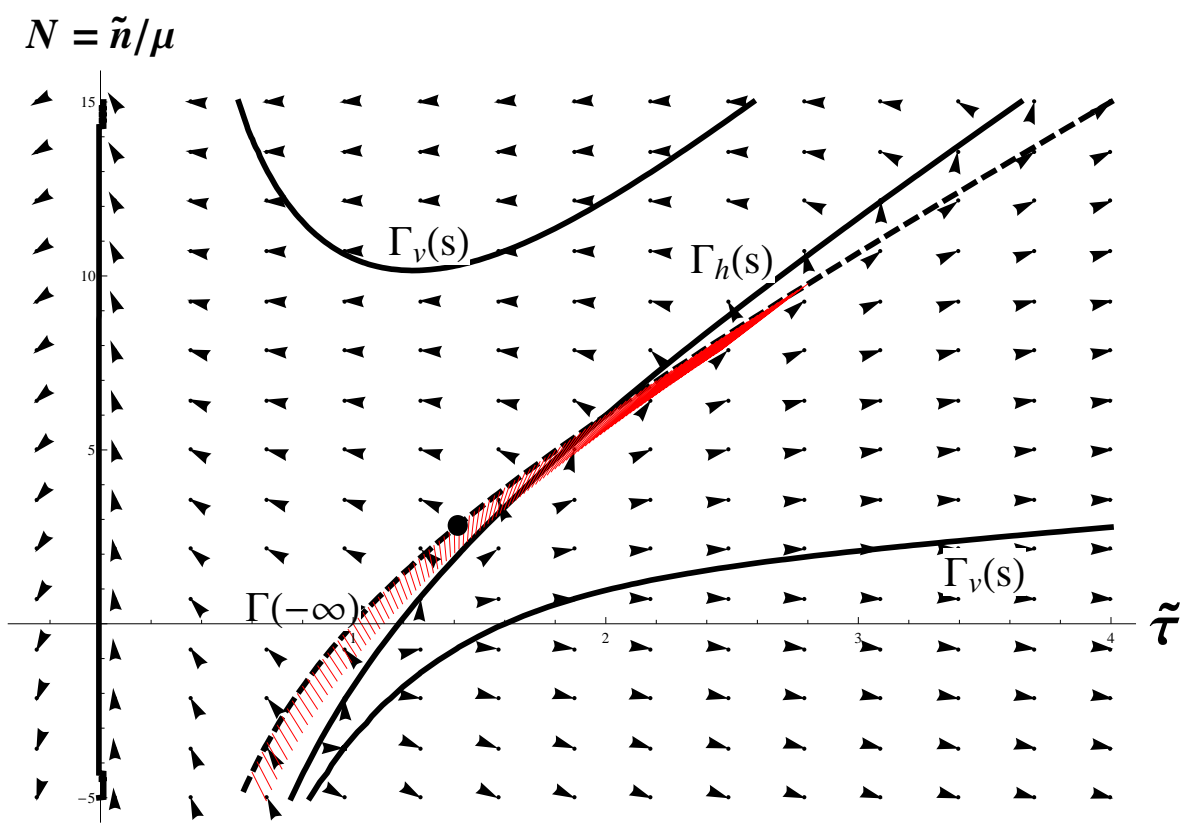

Figure 5: The vector field of the dynamical system run in reverse

Then $\Phi \in C^{1}(\Omega)$, i.e., $\Phi$ is continuously differentiable in both its $s$ and $\mathbf{y}_{0}$ arguments. See Section 2.5 of $[22]$.

Claim 3 At $s=1$, the positions of the solutions tracing out the unstable manifolds form a continuous curve in the region between $\Gamma(-\infty)$ and the lower branch of $\Gamma_{v}(1)$.

Proof of Claim 3 Fix $\widetilde{\tau}_{0}>0$. Define $\mathcal{E}$ by

$$
\mathcal{E}=\left\{(\widetilde{\tau}, N):(\widetilde{\tau}, N) \text { is between } \Gamma(-\infty) \text { and the lower branch of } \Gamma_{v}(1) \text {, and } \widetilde{\tau}>\widetilde{\tau}_{0}>0\right\}
$$

Then $\mathbf{f} \in C^{1}(\mathcal{E})$, which implies that the flow $\Phi$ of the dynamical system is also continuously differentiable. The Center Manifold Theorem (see Section 2.12 of [22]) guarantees the unstable manifolds can be locally (near the fixed points) continuously parametrized by $s$ and an arclength coordinate for the curve of fixed points. Hence for some $s_{0}>-\infty$ with $\left|s_{0}\right|>>1$ there is a continuous curve formed by the positions of the solutions that trace out unstable manifolds. See Figure 6.

Since $\mathbf{f} \in C^{1}(\mathcal{E})$, the maximal interval of existence for each unstable manifold includes the half-infinite interval $(-\infty, 1]$. Hence the continuous curve formed by the solutions at $s=s_{0}$ is mapped continuously via $\Phi$ to its image at $s=1$. By continuity of the $s=s_{0}$ curve and of $\Phi$, the positions of the solutions at $s=1$ also form a continuous curve.

Note that $\mathcal{E}$ is bounded below by $\widetilde{\tau}_{0}>0$. $\widetilde{\tau}_{0}$ can be re-defined to allow $\mathcal{E}$ to extend to any proximity to the $\widetilde{\tau}=0$ line, and the argument still holds. On the curve of fixed points, $N \rightarrow-\infty$ as $\widetilde{\tau} \rightarrow 0$, so the continuous curve of positions at $s=1$ is infinite in extent. This argument leaves open the possibility that the map from the fixed points to this curve may not be uniformly continuous. 


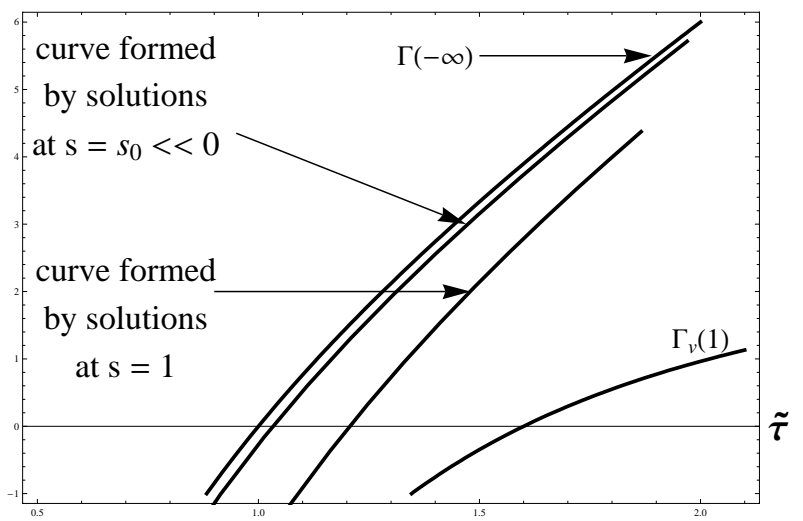

Figure 6: The continuity of the curve of fixed points is maintained in the curves formed by the unstable manifold orbits for $s>-\infty$.

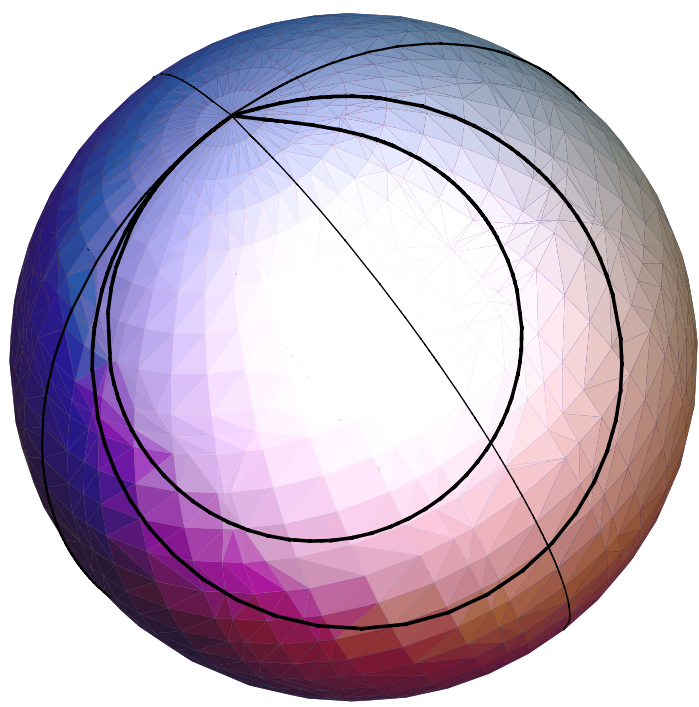

Figure 7: The stereographic projections of the lower branch of $\Gamma_{v}(1)$ (outer curve) and $\Gamma(-\infty)$ (inner curve) intersect at the north pole of the sphere. The dashed curve that they both intersect is the projection of the $N=0$ line. The curve of loci at $s=1$ is between these two curves and evolves into $\Gamma(-\infty)$ as $s \rightarrow-\infty$.

Claim 4 The continuous curve of positions of solutions at $s=1$ intersects the line $N=0$.

Sketch of Proof of Claim 4 This can be proved by considering the one-point compactification of the $(\widetilde{\tau}, N)$-plane, which is a 2-sphere, and examining the stereographic projections onto the sphere of $\Gamma(-\infty)$, the lower branch of $\Gamma_{v}(1)$, and the curve of solution positions at $s=1$. Each of these continuous images of $\mathbb{R}$ is projected to a closed loop on the sphere; see Figure 7 . Each "end" of the projections of $\Gamma(-\infty)$ and the lower branch of $\Gamma_{v}(1)$ lie at the north pole of the sphere. The closed loop of solution positions at $s=1$ lies between these projections. If the loop of positions is to evolve into $\Gamma(-\infty)$ as $s \rightarrow-\infty$, the "ends" of this loop must also lie at the north pole. Hence the loop of positions must intersect the $N=0$ line on the sphere, shown 
in Figure 7.

\subsection{Numerical Results}

We use the form of the fixed points of Eq. (66)-(68) to compute numerical solutions of the equations. The method is as follows.

1. Choose $\widetilde{\tau}_{0}>0$.

2. Set $N_{0}=(1+\kappa)\left(\widetilde{\tau}_{0}-\widetilde{\tau}_{0}^{-1}\right)$, the $N$-coordinate of the fixed point with $\widetilde{\tau}$-coordinate equal to $\widetilde{\tau}_{0}$.

3. Compute the unstable eigenvector at the fixed point $\left(\widetilde{\tau}_{0}, N_{0}, 1\right)$.

4. For some small $\epsilon>0$, perturb $\left(\widetilde{\tau}_{0}, N_{0}, 1\right)$ by $\epsilon$ times the unstable eigenvector:

$$
\left(\begin{array}{c}
\widetilde{\tau}_{1} \\
N_{1} \\
\left(\widetilde{\gamma}_{2}\right)_{1}
\end{array}\right)=\left(\begin{array}{c}
\widetilde{\tau}_{0} \\
N_{0} \\
1
\end{array}\right)+\epsilon\left(\begin{array}{c}
\left((1+\kappa) \widetilde{\tau}_{0}-2 \widetilde{\tau}_{0}^{3}\right) / 4\left(1+\kappa+\widetilde{\tau}_{0}^{2}\right) \\
-(1+\kappa)\left(2 \widetilde{\tau}_{0}^{4}+\left(3-\kappa^{2}\right) \widetilde{\tau}_{0}^{2}+(1+\kappa)\right) / 4\left(1+\kappa+\widetilde{\tau}_{0}^{2}\right) \\
1
\end{array}\right)
$$

5. Set $s_{1}=1+\frac{1}{2} \ln \left(\epsilon / b_{2}\right)$. The formula in the step above gives an approximation for the position, at $s=s_{1}$, of the solution tracing out the unstable manifold attached to the fixed point $\left(\widetilde{\tau}_{0}, N_{0}, 1\right)$.

6. Integrate the equations numerically (for increasing $s$ ) until $N=0$.

If $N$ reaches zero at some $s$-value other than 1 , adjust $\widetilde{\tau}_{0}$ and repeat the above steps until $|N(1)|$ is near zero to within some tolerance.

Results for $R_{\max }=1$ are pictured in Figures 8-13. In each case, the radial stress is zero at the periphery and positive at the center, as expected from the form of the vector field run in reverse; see Figure 5. The azimuthal stress, which agrees with the radial stress at $R=0$, is negative at the periphery, indicating that the outer edge is under azimuthal compression.

Recall that for $\kappa>1, \Gamma_{v}(s)$ has two branches. The impact of the change in the vector field can be seen by comparing Figures 9 and 11. As $R \rightarrow 0(s \rightarrow-\infty)$, the terminal $\tau$-value is approached from below in Figure 9, indicating that the fixed point is approached from the left. In Figure 11, on the other hand, the terminal $\tau$-value is approached from larger values of $\tau$, indicating that the fixed point is approached from the right.

\section{Constant Radial Growth: $\gamma_{1}(R) \equiv \gamma_{0}>1$}

Now we consider a growth pattern that allows us to use much of the previous section's analysis but produces quite different results. We again let the radial growth $\gamma_{1}$ be constant, but we assign it a value $\gamma_{0}$ greater than one. Further, we assume $\gamma_{2}(R) \leq \gamma_{0}$, with equality only at $R=0$. 


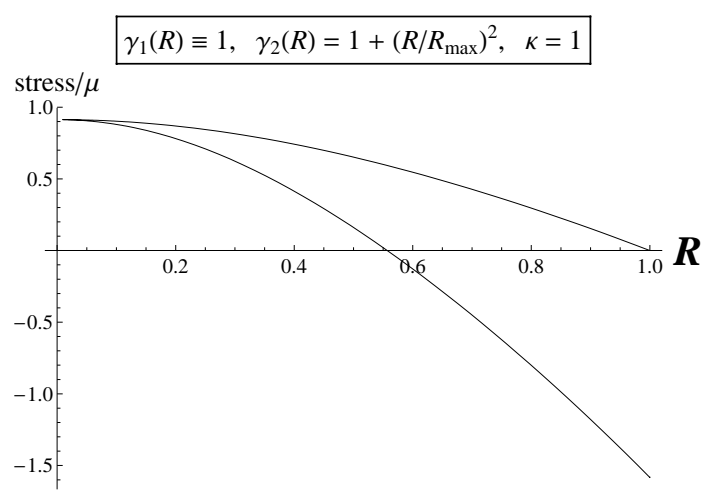

Figure 8: The radial stress (upper curve) drops to zero at the periphery, while the azimuthal stress (lower curve) is negative at the periphery.

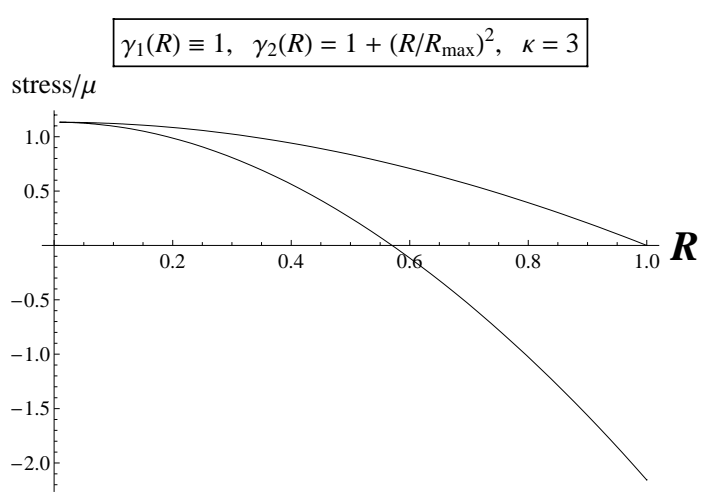

Figure 10: Changing $\kappa$ from 1 to 3 produces little change in the stress profiles.

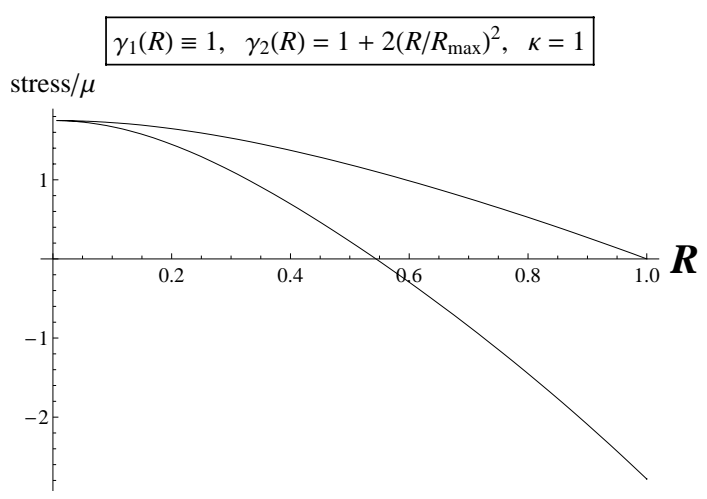

Figure 12: Changing $b$ from 1 to 2 produces little change in the stress profiles.

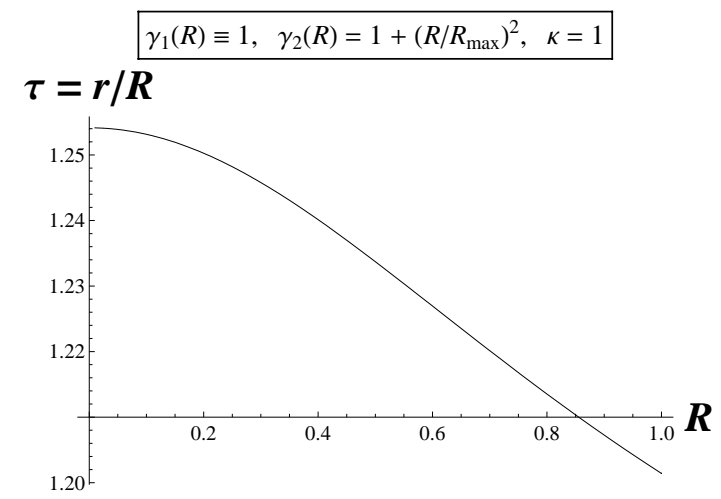

Figure 9: $\tau=r / R$ decreases monotonically in this configuration.

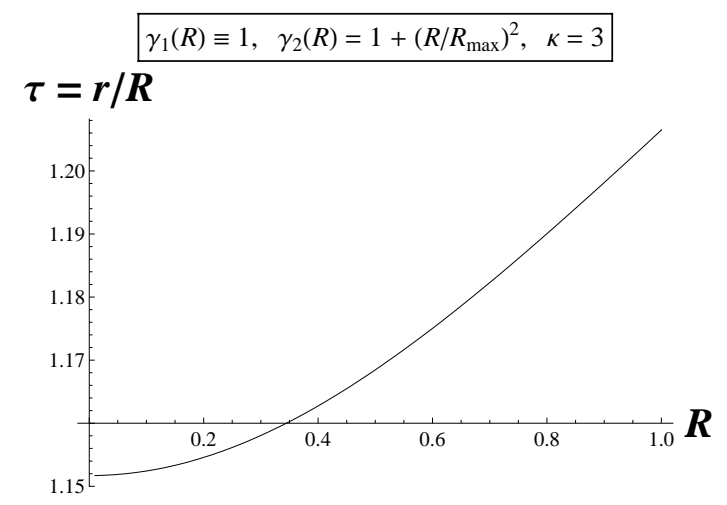

Figure 11: Changing $\kappa$ from 1 to 3 changes the $\tau=r / R$ profile dramatically.

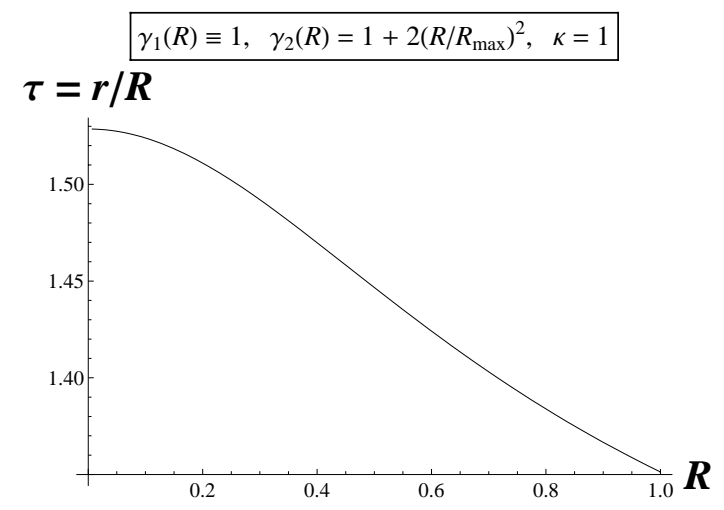

Figure 13: Changing $b$ from 1 to 2 produces little change in the $\tau=r / R$ profile.

$$
\text { If } \gamma_{1}(R) \equiv \gamma_{0}>1 \text { and } \gamma_{2}(R)=\gamma_{0}-b\left(R / R_{\max }\right)^{2} \text {, the corresponding differential equations }
$$


are

$$
\begin{aligned}
\frac{d N}{d s} & =\frac{\gamma_{0}}{\widetilde{\gamma}_{2}} \widetilde{\tau}+\frac{\kappa \gamma_{0}}{2 \widetilde{\gamma}_{2}}\left(N-\kappa \widetilde{\tau}+\sqrt{(N-\kappa \widetilde{\tau})^{2}+4(1+\kappa) \widetilde{\gamma}_{2}^{2}}\right)-\frac{(1+\kappa) \gamma_{0} \widetilde{\gamma}_{2}}{\widetilde{\tau}}-N, \\
\frac{d \widetilde{\tau}}{d s} & =\frac{\gamma_{0}}{2 \widetilde{\gamma}_{2}}\left(N-\kappa \widetilde{\tau}+\sqrt{(N-\kappa \widetilde{\tau})^{2}+4(1+\kappa) \widetilde{\gamma}_{2}^{2}}\right)-\widetilde{\tau} \\
\frac{d \widetilde{\gamma}_{2}}{d s} & =2\left(\widetilde{\gamma}_{2}-\gamma_{0}\right) .
\end{aligned}
$$

The fixed points of this system form curves whose coordinates satisfy

$$
\widetilde{\gamma}_{2}=\gamma_{0}, \quad \widetilde{\tau}=\frac{N \pm \sqrt{N^{2}+4(1+\kappa)^{2} \gamma_{0}^{2}}}{2(1+\kappa)} .
$$

$\widetilde{\tau}>0$ on one curve; $\widetilde{\tau}<0$ on the other. We focus on positive $\widetilde{\tau}$. At each such fixed point, the linearization $D \mathbf{f}$ is

$$
\left(\begin{array}{ccc}
-\frac{(1+\kappa)\left(\tilde{\tau}^{2}+\gamma_{0}^{2}\right)}{\tilde{\tau}^{2}+(1+\kappa) \gamma_{0}^{2}} & \frac{\tilde{\tau}^{2}}{\tilde{\tau}^{2}+(1+\kappa) \gamma_{0}^{2}} & \frac{\tilde{\tau}\left(-\tilde{\tau}^{2}+(1+\kappa) \gamma_{0}^{2}\right)}{\gamma_{0}\left(\tilde{\tau}^{2}+(1+\kappa) \gamma_{0}^{2}\right)} \\
\frac{(1+\kappa)\left(\tilde{\tau}^{2}+\gamma_{0}^{2}\right)\left(-(-1+\kappa) \tilde{\tau}^{2}+(1+\kappa) \gamma_{0}^{2}\right)}{\tilde{\tau}^{2}\left(\tilde{\tau}^{2}+(1+\kappa) \gamma_{0}^{2}\right)} & \frac{(-1+\kappa) \tilde{\tau}^{2}-(1+\kappa) \gamma_{0}^{2}}{\tilde{\tau}^{2}+(1+\kappa) \gamma_{0}^{2}} & \frac{-(1+\kappa) \tilde{\tau}^{4}+\left(-2-\kappa+\kappa^{2}\right) \tilde{\tau}^{2} \gamma_{0}^{2}-(1+\kappa)^{2} \gamma_{0}^{4}}{\tilde{\tau} \gamma_{0}\left(\tilde{\tau}^{2}+(1+\kappa) \gamma_{0}^{2}\right)} \\
0 & 0 & 2
\end{array}\right)
$$

which has eigenvalues 2,0 , and -2 , regardless of $\kappa, \gamma_{0}$, and $\widetilde{\tau}$. As in the previous case, the only eigenvector with nonzero $\widetilde{\gamma}_{2}$-component is the unstable eigenvector, which in this case is

$$
\left(\frac{\tilde{\tau}\left(-2 \tilde{\tau}^{2}+(1+\kappa) \gamma_{0}^{2}\right)}{4 \gamma_{0}\left(\tilde{\tau}^{2}+(1+\kappa) \gamma_{0}^{2}\right)}, \frac{-2(1+\kappa) \tilde{\tau}^{4}+\left(-3-2 \kappa+\kappa^{2}\right) \tilde{\tau}^{2} \gamma_{0}^{2}-(1+\kappa)^{2} \gamma_{0}^{4}}{4 \tilde{\tau} \gamma_{0}\left(\tilde{\tau}^{2}+(1+\kappa) \gamma_{0}^{2}\right)}, 1\right)^{T} .
$$

In this example, $\widetilde{\gamma}_{2}(s) \uparrow \gamma_{0}$ as $s \rightarrow-\infty$, which indicates that the unstable manifold approaches the fixed point from the direction opposite to this eigenvector; see Figure 14.

As in the previous section, we define horizontal and vertical isoclines $\Gamma_{h}(s)$ and $\Gamma_{v}(s)$, respectively, and observe that they coincide with $\Gamma(-\infty)$, the curve of fixed points, as $s \rightarrow-\infty$. Arguments similar to those in the last section show the existence of an unstable manifold that intersects the line $N=0$ at $s=1$. In this case, however, it is possible for a solution tracing out an unstable manifold to satisfy $N(1)=0$ as well as $\lim _{s \rightarrow-\infty} N(s)<0$, which corresponds to compressive radial stress at the center of the disc.

Using a numerical approach nearly identical to that of the last section (with negative $\epsilon$ in step 4), we found results with negative radial stress throughout the body and azimuthal stress that changes sign within the body. In these examples, $\gamma_{0} \geq \gamma_{2}(R) \geq 1$, with $\gamma_{2}(0)=\gamma_{0}$ and $\gamma_{2}\left(R_{\max }\right)=1$.

This type of growth tensor induces negative radial stress throughout the body; it is largest at $R=0$ and decays monotonically until it is zero at the periphery. At $R=0$, the azimuthal stress agrees with the radial stress. It grows monotonically in $R$ and is positive at the periphery. The presence of negative stresses through so much of the body suggests that these configurations may be prone to buckling. 


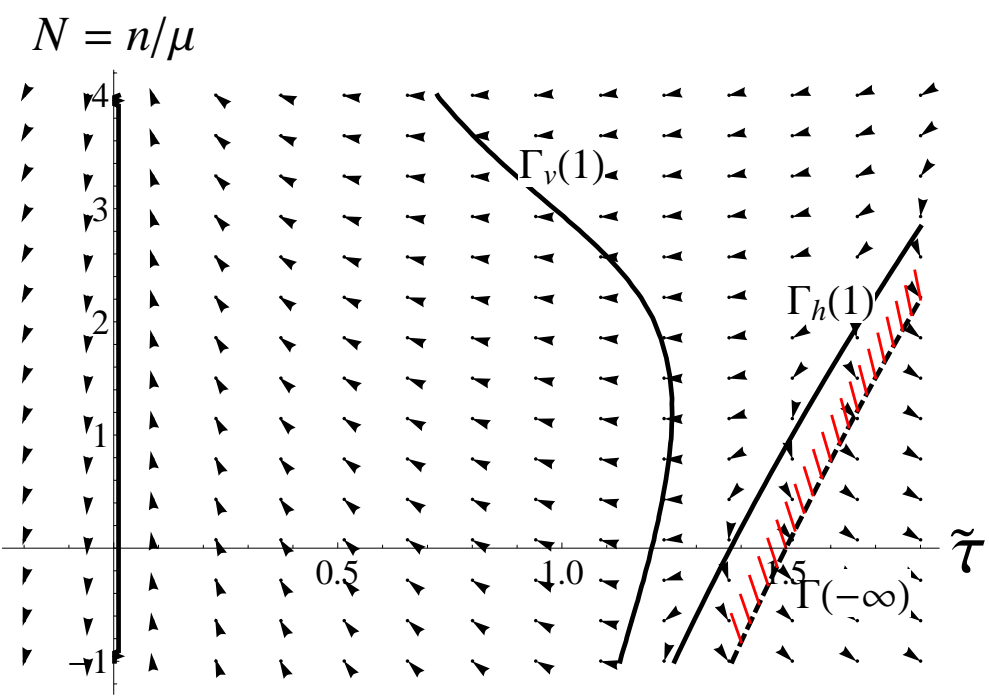

Figure 14: The vector field of the dynamical system run in reverse. In this case, the solutions tracing out the unstable manifolds approach the fixed points from the left as $s \rightarrow-\infty$.

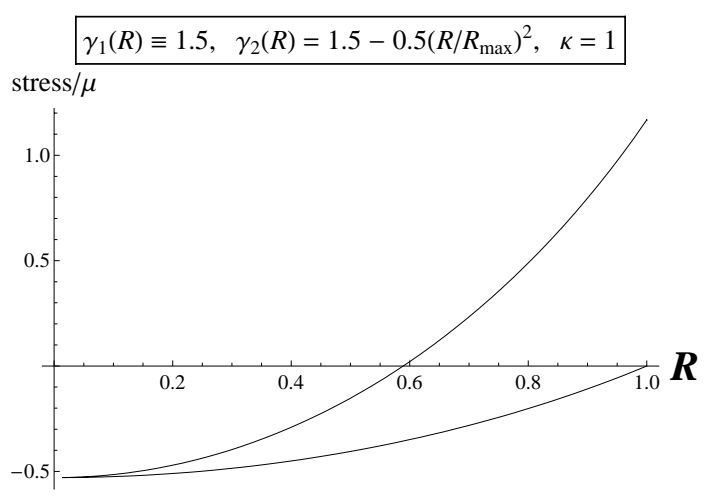

Figure 15: The radial stress is negative throughout the body, while the azimuthal stress changes sign, leaving it negative at the periphery.

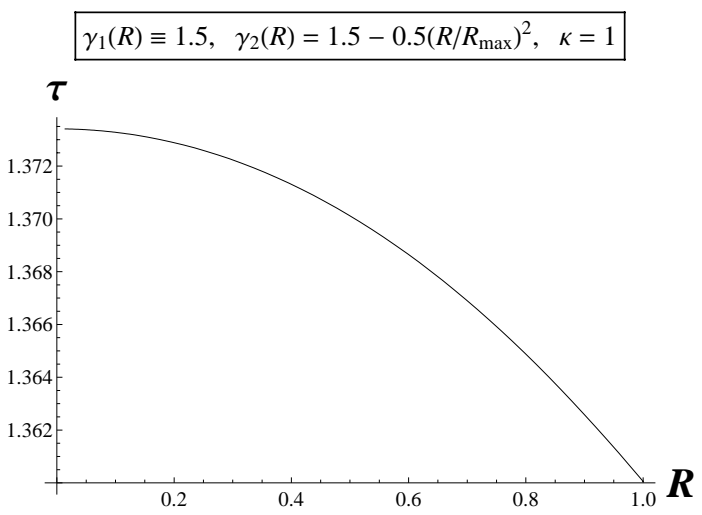

Figure 16:

\section{Conclusion}

Previously, the impact of the multiplicative decomposition in nonlinearly elastic bodies has been explored in detail for cylindrical and spherical shells and for cylindrical columns. Here we have shown how to produce a rigorous model of a globally constrained, incompatibly grown, three-dimensional plate with nonlinearly elastic behavior. This was accomplished by adopting the multiplicative decomposition of the deformation gradient and using the elastic portion of this decomposition as the argument of the constitutive relation. We have employed the Global Constraint Principle to derive balance laws for cylindrical deformations of an axisymmetric plate and combined them with the modified constitutive relation.

Using a compressible neo-Hookean constitutive relation, we derived a complete set of ordinary differential equations and boundary conditions to describe the final configuration. A 


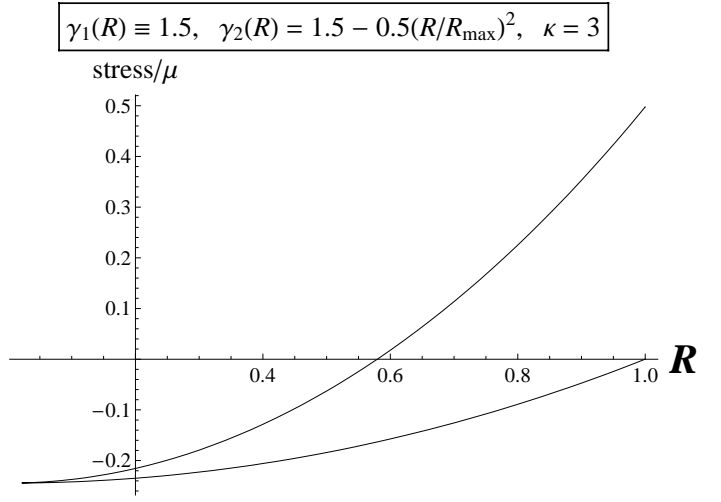

Figure 17: Changing $\kappa$ from 1 to 3 changes the magnitudes of the stresses.

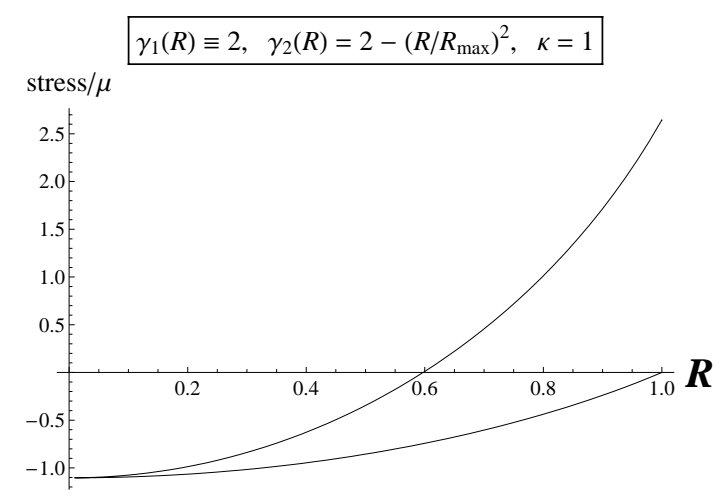

Figure 19: Keeping $\kappa=1$ but doubling the growth approximately doubles the stress.

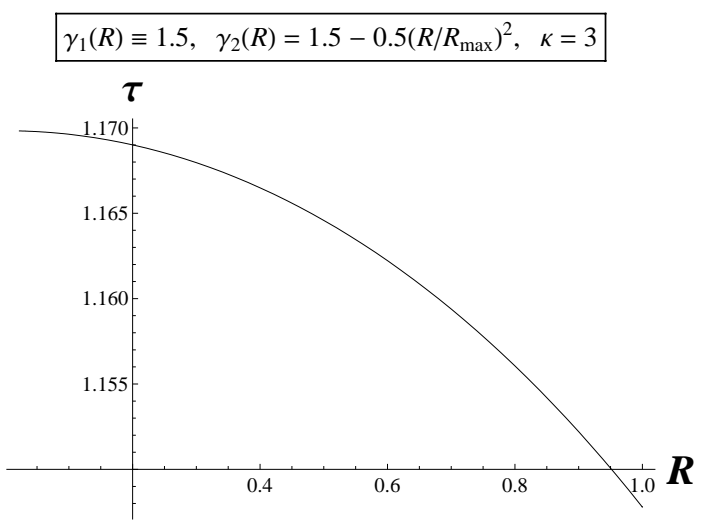

Figure 18: Unlike what was found in the previous case, changing $\kappa$ from 1 to 3 has little impact on the form of $\tau=r / R$.

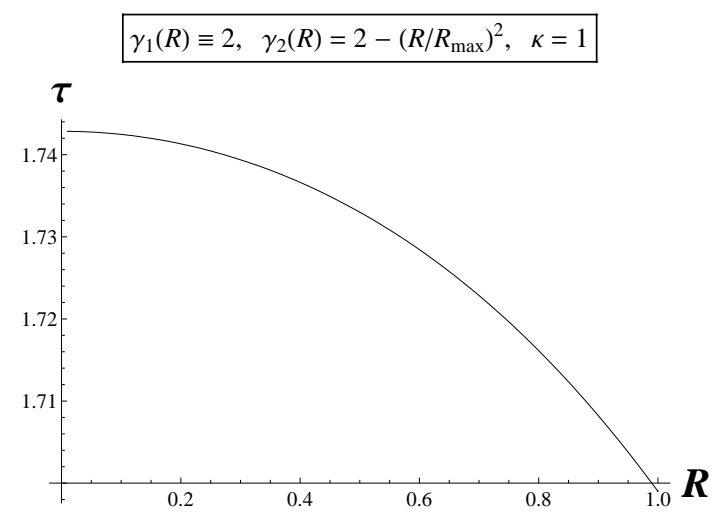

Figure 20: Keeping $\kappa=1$ but doubling the growth approximately doubles the expansion.

change of variable produced an equivalent set of autonomous equations. For various choices of growth tensor, we have used basic results of dynamical systems theory to demonstrate the existence of a final configuration of an unloaded plate that carries residual stress due to incompatible growth.

Adjustment of the growth tensors produced solutions featuring a variety of stress and strain profiles. In cases in which the radial growth is larger than the azimuthal growth, the radial residual stress is compressive within the body, while the azimuthal residual stress is compressive at the center of the body and tensile at the periphery. Large enough compressive stress can lead to buckling in nonlinearly elastic plates. Therefore, it is reasonable to expect that suitable growth profiles will generate sufficient compressive residual stress to create instabilities of the kind found in nonlinearly elastic spherical shells $[1,14]$. A detailed analysis should reveal which growth tensors induce such an instability.

Acknowledgments: This publication is based on work supported by Award No. KUK-C1013-04, made by King Abdullah University of Science and Technology (KAUST), and based 
in part upon work supported by the National Science Foundation under grants DMS-0907773 $(\mathrm{AG}, \mathrm{MT})$

\section{References}

[1] M. Ben Amar and A. Goriely. Growth and instabilities in elastic tissues. Journal of the Mechanics and Physics of Solids, 53, 2005.

[2] D. Ambrosi, A. Guillou, and E. S. Di Martino. Stress-modulated remodeling of a nonhomogeneous body. Biomechanics in Modeling and Mechanobiology, 7, 2008.

[3] S. S. Antman. Nonlinear Problems of Elasticity. Springer, Berlin, 1995.

[4] S. S. Antman and R. S. Marlow. Material constraints, Lagrange multipliers, and compatibility. Archive for Rational Mechanics and Analysis, 116:257-299, 1991.

[5] F. Brauer and J. A. Nohel. The Qualitative Theory of Ordinary Differential Equations. Dover Publications, New York, 1989.

[6] L. Cardamone, A. Valentín, JF Eberth, and JD Humphrey. Origin of axial prestretch and residual stress in arteries. Biomechanics and modeling in mechanobiology, 2009.

[7] H. R. Chaudhry, B. Bukiet, A. Davis, A. B. Ritter, and T. Findley. Residual stresses in oscillating thoracic arteries reduce circumferential stresses and stress gradients. Journal of biomechanics, 30(1):57-62, 1997.

[8] Y.-C. Chen and A. Hoger. Constitutive functions of elastic materials in finite growth and deformation. Journal of Elasticity, 59:173-193, 2000.

[9] P. G. Ciarlet. An Introduction to Differential Geometry with Applications to Elasticity. Springer, Dordrecht, 2005.

[10] A. Delfino, N. Stergiopulos, J. E. Moore, and J. J. Meister. Residual strain effects on the stress field in a thick wall finite element model of the human carotid bifurcation. $J$. Biomech., 30(8):777-786, 1997.

[11] J. Dervaux, P. Ciarletta, and M. Ben Amar. Morphogenesis of thin hyperelastic plates: A constitutive theory of biological growth in the Föppl-von Kármán limit. Journal of the Mechanics and Physics of Solids, 57(3):458-471, 2009.

[12] E. Efrati, E. Sharon, and R. Kupferman. Elastic theory of unconstrained non-Euclidean plates. Journal of the Mechanics and Physics of Solids, 57:762-775, 2009.

[13] Y. C. Fung. What are the residual stresses doing in our blood vessels? Annals of Biomedical engineering, 19(3):237-249, 1991. 
[14] A. Goriely and M. Ben Amar. Differential growth and instabilities in elastic shells. Physical Review Letters, 94:1918103, 2005.

[15] A. Goriely, R. Vandiver, and M. Destrade. Nonlinear Euler buckling. Proceedings of the Royal Society of London Series A, 464, 2008.

[16] A. Gupta, D. J. Steigmann, and J. S. Stölken. On the evolution of plasticity and incompatibility. Mathematics and Mechanics of Solids, 12:583-610, 2007.

[17] E. Kroner. Alleganeine kontinuumstheorie der verzetsmugen and eigenspannuugen. Archive for Rational Mechanics and Analysis, 4:273-334, 1960.

[18] M. LeBret. Catastrophic variations of twist and writhing of circular DNA with constraint? Biopolymers, 18, 1979.

[19] R. S. Marlow. Global material constraints and their associated reactive stresses. Journal of Elasticity, 33:203-212, 1993.

[20] J. McMahon, A. Goriely, and M. Tabor. Spontaneous Cavitation in Growing Elastic Membranes. Mathematics and Mechanics of Solids, page doi:10.1177/1081286508092010, 2008 .

[21] R. W. Ogden. Non-Linear Elastic Deformations. Dover, Mineola, NY, 1984.

[22] L. Perko. Differential Equations and Dynamical Systems, 2nd ed. Springer, New York, 1996.

[23] E. K. Rodriguez, A. Hoger, and A. D. McCulloch. Stress-dependent finite growth law in soft elastic tissue. Journal of Biomechanics, 27:455-467, 1994.

[24] L. A. Taber and J. D. Humphrey. Stress-modulated growth, residual stress, and vascular heterogeneity. Transactions of the ASME, 123:528-535, 2001.

[25] R. N. Vaishnav and J. Vossoughi. Residual stress and strain in aortic segments. J Biomech, 20(3):235-239, 1987.

[26] R. Vandiver and A. Goriely. Tissue tension and axial growth of cylindrical structures in plants and elastic tissues. Europhys. Lett. (EPL), 84(58004), 2008.

[27] H. Xiao, T. O. Bruhns, and A. Meyers. Elastoplasticity beyond small deformations. Acta Mechanica, 182:31-111, 2006. 



\section{RECENT REPORTS}

36/09 Modelling bacterial behaviour close to a no-slip plane boundary: Shum the influence of bacterial geometry

37/09 Optimal L2-error estimates for the semidiscrete Galerkin approx-

Goswami imation to a second order linear parabolic initial and boundary value problem with nonsmooth initial data

38/09 Optimal L2 estimates for semidiscrete Galerkin methods for parabolic integro-differential equations with nonsmooth data

Pani

Goswami

Pani

Yadav

39/09 Spatially structured oscillations in a two-dimensional excitatory neuronal network with synaptic depression

Kilpatrick

Bressloff

40/09 Stationary bumps in a piecewise smooth neural field model with synaptic depression

Kilpatrick

Bressloff

41/09 Homogenization for advection-diffusion in a perforated domain Haynes

Hoang

Norris

Zygalakis

42/09 Fast stochastic simulation of biochemical reaction systems by alternative formulations of the Chemical Langevin Equation

Melykuti

Burrage

Zygalakis

43/09 Pseudoreplication invalidates the results of many neuroscientific

Lazic studies

44/09 Cardiac cell modelling: Observations from the heart of the cardiac physiome project

45/09 A Hybrid Radial Basis Function - Pseudospectral Method for Thermal Convection in a 3-D Spherical Shell

Wright

Flyer

46/09 Refining self-propelled particle models for collective behaviour Yates

Baker

Erban

Maini

47/09 Stochastic Partial Differential Equations as priors in ensemble methods for solving inverse problems

Potsepaev

Farmer

Aziz

48/09 DifFUZZY: A fuzzy spectral clustering algorithm for complex data

Cominetti et al. sets

01/10 Fluctuations and instability in sedimentation

Guazzelli

Hinch 
02/10 Determining the equation of state of highly plasticised metals from

Hinch boundary velocimetry

03/10 Stability of bumps in piecewise smooth neural

Kilpatrick elds with nonlinear adaptation

Bressloff

04/10 Random intermittent search and the tug-of--war model of motor-

Newby driven transport

Bressloff

05/10 Ergodic directional switching in mobile insect groups

Escudero et al.

06/10 Derivation of a dual porosity model for the uptake of nutrients by root hairs

Zygalakis

Roose

07/10 Frost heave in compressible soils

Majumdar

Peppin

Style

Sander

08/10 A volume-preserving sharpening approach for the propagation of

Reis sharp phase boundaries in multiphase lattice Boltzmann simulations

09/10 Anticavitation and differential growth in elastic shells

Moulton

Goriely

10/10 On the mechanical stability of growing arteries

Goriely

Vandiver

11/10 Nonlinear Correction to the Euler Buckling Formula for Compressible Cylinders

De Pascalis

Destrade

Goriely

Copies of these, and any other OCCAM reports can be obtained from:

Oxford Centre for Collaborative Applied Mathematics

Mathematical Institute

24 - 29 St Giles'

Oxford

OX1 3LB

England

www.maths.ox.ac.uk/occam 